\title{
1995 MRS Fall Meeting
}

$T_{1}$ turing about 4,000 oral and poster presentations. For the second year, the meeting will include the Sheraton Boston Hotel in addition to the Boston Marriott and Westin Hotels. There will be four evenings of poster sessions, and the Exhibit (Tuesday through Thursday) has expanded into all three hotels and includes a Wednesday evening session.

A new tutorial program debuts at this meeting which integrates half- and fullday tutorials into the technical program of selected symposia. These tutorials are scheduled Sunday through Tuesday, and are listed in the following pages and in the Meeting Program.

\section{Technical Features}

Several symposia address electronic materials, with an emphasis on wide bandgap semiconductors and electronic ceramics. Symposium AAA will feature the latest results in the very rapidly growing field of group III-nitrides, highlighting achievements in diode laser heterostructures and in InGaN and GaN/SiC LEDs. Symposium DD will cover electronic applications of diamond and diamond-like materials, including their use as cathodes in field emission flat panel displays, sensing applications, and switching devices. Symposia $G$ and BB address oxide and ceramic thin films for a variety of electronic applications. The formation of quantum wires by strain-induced lateral-layer ordering will be one of the topics on optoelectronic materials covered in Symposium EE, with potential applications for quantum wire lasers.

Ion beam technology will be featured in Symposium A, which will include sessions on surface optical property modifications, computer simulations of energetic particle/surface interactions, and the formation of nanocrystals and quantum dots in insulators by ion implantation. A summary of a DOE panel study, Energetic Ion Beams in Semiconductor Processing, is also planned.

The areas of thin film growth and processing will be covered in several symposia which will individually focus on laser processing, phase transformations, epitaxial structure, and metastable phases. In Symposium I, a technique using orientation imaging will be described for determining grain orientations, an important issue for magnetic disk storage media.

The development of diagnostic techniques for semiconductor materials processing and characterization techniques for probing surfaces and interfaces will be represented in several symposia ( K, L, CC, and J). These include advances in in situ techniques which make it possible to observe electromigration induced void dynamics in metal interconnects, as well as
November 27-December 1, 1995 Boston Marriott Hotel and Westin Hotel/Copley Place Sheraton Boston Hotel Boston, Massachusetts

\author{
Meeting Chairs: \\ Michael J. Aziz, \\ Harvard University \\ Berend T. Jonker, \\ Naval Research Laboratory \\ Leslie J. Struble, \\ University of Illinois-Urbana
}

new techniques which can probe the dynamics of gas-solid surface interactions at the atomic level.

The study of crystal defects continues to be an underlying theme of high- $\mathrm{T}_{\mathrm{c}}$ superconductor research. The nature of defects in these materials and their role in determining microscopic and macroscopic properties will be explored in Symposium $\mathrm{F}$, with several joint sessions planned with Symposium G.

Symposium $G$ will address several classes of epitaxial oxide thin films, and will include presentations on a new class of lanthanum manganate materials with large magnetoresistive properties, which have possible applications in thin-film heads for magnetic data storage.

A wide variety of topics in the areas of organic and polymeric materials will be covered in Symposia W and Y. Symposium $\mathrm{W}$ will address electrical, optical, and magnetic properties, while Symposium $Y$ will focus on long-term performance issues, such as the in-service weatherability of plastics and coatings and the physical aging of polymers.

Several symposia cover topics in the biological sciences and other complex or "soft" material systems. Symposium U will consider how lessons learned in nature may be applied to develop new materials, and Symposium $\mathrm{Z}$ addresses the development of materials suitable for interfacing with human tissue, as may be encountered in implants or joint replacements. Symposium O will describe how self-assembled materials and micron- to submicron-sized arrays have been developed as substitutes for gel electrophoresis to fractionate DNA. Symposium N covers a variety of topics related to foams, emulsions, colloids, and liquid crystals.

Symposium $\mathrm{P}$ will provide a representative picture of how computer simulations are complementing, and in some cases replacing real experiments in materials research, making it possible to obtain insight of unprecedented detail and depth. The power of massively parallel computers to study fractures will be demonstrated in Symposium Q. Molecular dynamics simulation techniques can model the time evolution of 106 to 108 atoms, several orders of magnitude beyond what was possible only a few years ago, providing new capabilities to model ductile and brittle fracture of crystals at the atomic scale.

Symposium $\mathrm{R}$ emphasizes the relationship between microstructure and chemical degradation of cement-based systems. Topics include the transport of aggressive ions in limiting the rate of chemical attack and the changes in the microstructure due to this attack.

As part of Symposium V, Richard L. Garwin, IBM Fellow Emeritus who has been involved in science policy issues relating to arms control and nuclear war, will discuss the disposition of excess weapons-grade plutonium. Other speakers in this symposium will address issues such as treatment alternatives to incineration, and mineral studies that reveal the types of alteration that may occur due to repository emplacement.

Various symposia also cover energetic materials; low-cost manufacturing; silicide thin films; fullerenes and buckytubes; electrically based microstructural characterization; and Frontiers of Materials Research, which provides reviews for the nonspecialist in areas of active research

\section{Special Features}

The plenary speaker Monday night will be Robert Walker, chair of the Committee on Science in the U.S. House of Representatives, whose jurisdiction includes space and aeronautics, basic research, energy and environment research and development, and technology. This talk will set the stage for a panel on the Changing Character of Science Policy, moderated by D. Allan Bromley on Tuesday at 8:00 a.m.

A workshop on materials education will be held Thursday, including an open lunchtime forum on materials curricula.

Recipients of the Von Hippel Award, the MRS Medals, the Turnbull Lectureship, and the graduate student awards will be honored at a Wednesday evening awards ceremony. Immediately following the ceremony, the Von Hippel Award recipient will give a presentation, and a reception will follow. Talks by the other award recipients have been integrated into the technical program. See the following pages and next month's MRS Bulletin for more information on award recipients and their presentations.

Other events include a student mixer and a Women in MRS meeting.

For further details about the meeting see the following pages and the 1995 MRS Fall Meeting Program, which has been mailed to all MRS members and is available on the MRS homepage (http://www.mrs. org/). To obtain a copy of the program or to register, contact MRS at (412) 367-3003; fax (412) 367-4373; or e-mail info@mrs.org. 


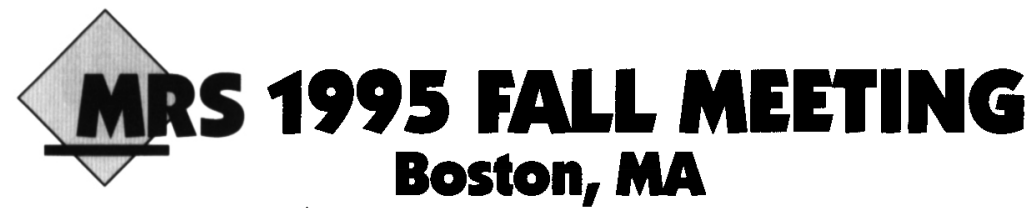 \\ Meeting Activities}

\section{Plenary Session}

Robert S. Walker, U.S. Congressman

Monday, November $27 \bullet 6: 00$ p.m., Salon E • Boston Marriott

Forums

\section{The Changing Character of Science Policy}

Tuesday, November 28, 8:00-9:00 a.m.

Boston Marriott - Salon F

Moderator: D. Allan Bromley

Panelists: (to be announced)

With the recent rapid change in university, industrial, and government research environments due to such factors as limited funding, global competition, government agency restructuring, and the refocusing of government missions, there is widespread interest among scientists and engineers in understanding and responding to new realities. The long-lasting impact on when, where and how future research is conducted will be the focus of the forum speakers followed by an open discussion.

\section{- Workshop on Materials Education}

Thursday, November 30 - Boston Marriott

8:30-10:30 a.m. Invited Presentations and Discussion Boston University Room

10:30-noon Lab/PC Demonstration, Displays, and Posters Simmons Room

noon-1:30 p.m. Lunchtime Forum on "Materials Curricula" Boston University Room

(Box lunches available in the Exhibit Hall)

1:30-5:00 p.m. Invited Presentations

Boston University Room

\section{Tumbull Lectureship}

Didier de Fontaine

University of California, Berkeley

Department of Materials Science and Engineering

Monday, November 27

Symposium P: Materials Theory, Simulations, and Parallel Algorithms, Session P2.1

10:15 a.m., Essex South

Westin Hotel

The Turnbull Lectureship Award will be presented to de Fontaine on Wednesday evening, November 29, Salon E, Boston Marriott.

\section{MRS Medal Awards}

\section{R.M. Tromp \\ IBM T.J. Watson Research Center}

Tromp will present his talk, "Si(001) Homoepitaxial Growth," on Thursday, November 30, at 9:00 a.m., in Symposium J: In-Situ Electron and Tunneling Microscopy of Dynamic Processes, Session J6.1, in the Wellesley Room, Boston Marriott.

\section{Federico Capasso}

AT\&T Bell Laboratories

Capasso will present his talk, "Bandgap Engineering of Compositionally Graded Semiconductors: Physics and Applications to Electronics and Photonics," on Thursday, November 30, at 1:30 p.m., in Symposium CC: Spectroscopy of Heterojunctions, Session CC7.1, in the Yarmouth/Vineyard Room, Boston Marriott.

\section{Women at MRS}

Tuesday, November 28, 7:00 - 8:00 a.m. (Check the Meeting Guide for details.)

\section{Job Placement}

A Job Placement Center will be conducted at the 1995 Fall Meeting of the Materials Research Society by the American Institute of Physics.

The Job Placement Center, including interviewing space, posting and call boards, will be open from 8:00 a.m. - 5:00 p.m., Tuesday, November 28, through Thursday, November 30, Boston Marriott. You need not attend, however, to have your résumé included on file.

Fees: None for MRS Members and Meeting Attendees

To register, or for more information, contact:

Career Planning \& Placement

American Institute of Physics

One Physics Ellipse

College Park, MD 20740

Phone: (301) 209-3190 - Fax: (301) 209-0841

Information and application forms are also available on the MRS Homepage (http://www.mrs.org/).

\section{Career Workshop}

A career workshop is scheduled for Sunday afternoon, November 26 (time and location to be announced), in conjunction with the 1995 Fall Meeting. Topics are expected to include: how to market your MS\&E education and experience; how to network successfully; and effective résumés and interviews.

Date, location, and program details to be announced.

For more information, contact:

MRS Member Services, Materials Research Society

9800 McKnight Road, Pittsburgh, PA 15237

Phone: (412) 367-3004, dial 1, ext. 400, \#

Fax: (412) 367-4373

e-mail: info@mrs.org

MRS Homepage: http://www.mrs.org/

\section{- Student Mixer}

All graduate students and members of MRS University Chapters are invited to attend a reception (date/time/location to be announced). Student chapters are a vital part of MRS, providing discussion between students and faculty and promoting student interest in materials science. Don't miss this opportunity to meet with others involved in MRS student activities.

\section{- Symposium Aide Positions}

Graduate students who plan to attend the 1995 Fall Meeting and are willing to assist in the symposium presentations by operating audio-visual equipment are encouraged to apply for a Symposium Aide position. By assisting in a minimum of four half-day sessions, aides will earn a waiver of the student registration fee, a complimentary full-year MRS student membership (January 1 through December 31,1996), and a small stipend to help defray expenses. Symposium preferences are assigned on a firstcome, first-serve basis. To request an application form and/or information, contact MRS Headquarters, (412) 367-3004 • Fax (412) 367-4373, E-mail: info@mrs.org. 


\section{Location/Lodging/Travel}

Boston Marriott/Copley Place 110 Huntington Avenue Boston, MA 02116

(800) 228-9290

(617) 236-5800 (Direct)

Fax (617) 236-5885

Rate: $\$ 120 /$ Single \$134/Double
Westin Hotel/Copley Place 10 Huntington Avenue Boston, MA 02116 (800) $228-3000$ Fax (617) 424-7483

Rate: \$124/Single \$144/Double (617) 262-9600 (Direct)

Sheraton Boston Hotel and Towers

Prudential Plaza

39 Dalton Street

Boston, MA 02199

(617) 236-2000 (Main Desk)

(617) 236-2020 (Reservations)

Fax (617) 236-1702

Rate: \$112/Single \$122/Double

\section{DEADLINE FOR HOTEL RESERVATIONS : NOVEMBER 1, 1995}

Blocks of rooms have been reserved for MRS meeting attendees at the Boston Marriott, Westin, and Sheraton Boston Hotels. Call your preferred hotel and refer to the Materials Research Society's meeting to receive the special rates.

A list of alternate lodging accommodations can be obtained via e-mail (info@mrs.org) or the MRS Homepage (http://www.mrs.org/).

\section{Airline Transportation}

The official travel management company for the Materials Research Society's 1995 Fall Meeting is Giselle's Travel Bureau. They will guarantee the lowest fares on any airline at time of booking. Call Giselle's, 1-800-523-0100, and mention the Materials Research Society's meeting, Monday Friday, 7:00 a.m. - 5:30 p.m. PST, or fax (916) 565-0936. MRS meeting attendees receive the following travel benefits and services:

Lowest fares on any airline guaranteed $\bullet$ Free flight insurance of $\$ 100,000$ - Vouchers for discounts on vacation packages - Computerized driving instructions from Logan International Airport to your hotel - Car rental savings

\section{Local Transportation}

Shuttle service to the Boston Marriott, Westin, and Sheraton Boston Hotels from Logan International Airport departs every half-hour, 7:00 a.m. - 7:00 p.m., from the designated shuttle stop in front of each terminal. The cost is approximately $\$ 7.50$ one way. Cab fares range between $\$ 15-\$ 18$ per ride (up to four persons can share one cab). There is a free shuttle from airport terminals to the airport subway station (The "T"). Copley Station is within one block of the Marriott, Westin, and Sheraton Hotels on the "Green Line."

\section{- Parking}

A parking garage is adjacent to the hotels at a daily cost of approximately $\$ 21$.

- Child Care

Check with the Concierge Desk at the individual hotels for a comprehensive roster of licensed and bonded sitters.

\section{All New! MRS 1995 Fall Meeting Tutorial Program}

\begin{tabular}{|c|c|c|}
\hline $\begin{array}{c}\text { SUNDAY } \\
\text { November } 26\end{array}$ & $\begin{array}{c}\text { MONDAY } \\
\text { November } 27\end{array}$ & $\begin{array}{c}\text { TUESDAY } \\
\text { November } 28\end{array}$ \\
\hline \multirow[t]{2}{*}{$\begin{array}{l}\text { Symposium G } \\
\text { 8:30 a.m. }-4: 30 \text { p.m. } \\
\text { Epitaxial Metal Oxide Thin Films } \\
\text { and Heterostructures }\end{array}$} & $\begin{array}{c}\text { Symposium J } \\
\text { 8:30 a.m. - 12:00 p.m. } \\
\text { In Situ Electron Microscopy: } \\
\text { Methodology \& Range of Applications }\end{array}$ & \\
\hline & $\begin{array}{c}\text { Symposium K } \\
\text { 8:30 a.m. - 12:00 p.m. } \\
\text { Effects Of Strain on Elec. \& Vibrational } \\
\text { Properties Of Semiconductors \& } \\
\text { Semiconductor Microstructures }\end{array}$ & \\
\hline $\begin{array}{c}\text { Symposium W } \\
\text { 1:30 p.m. - 5:00 p.m. } \\
\text { Electrical, Optical and Magnetic } \\
\text { Properties of Organic Solids }\end{array}$ & $\begin{array}{c}\text { Symposium Q } \\
\text { 8:30 a.m. }-12: 00 \text { p.m. } \\
\text { Introduction to Fracture: Brittle/Ductile } \\
\text { Transition \& Dynamic Instabiities in } \\
\text { Crack Properties }\end{array}$ & $\begin{array}{c}\text { Symposium CC } \\
\text { 1:30 p.m. }- \text { 5:00 p.m. } \\
\text { Novel Materials Research } \\
\text { Opportunities at National Synchotron } \\
\text { \& Free Electron Laser Faciities }\end{array}$ \\
\hline $\begin{array}{c}\text { Symposium X } \\
\text { 1:30 p.m. }-5: 00 \text { p.m. } \\
\text { Federal Materials Research Programs } \\
\text { and Opportunities }\end{array}$ & $\begin{array}{c}\text { Symposium H } \\
\text { 1:30 p.m. - 5:00 p.m. } \\
\text { Silicides for Integrated Circuits: } \\
\text { Materials Aspects \& Technical } \\
\text { Implementation }\end{array}$ & $\begin{array}{c}\text { Symposium DD } \\
\text { 1:00 p.m. - 5:00 p.m. } \\
\text { Electron Emission from Diamond }\end{array}$ \\
\hline
\end{tabular}


MAIL Return this form with payment to: Materials Research Society Meeting Registration

9800 McKnight Road

Pittsburgh, PA 15237-6006
PHONE Call the MRS Meeting Registration Desk (412) 367-3004, between 8:00 a.m. and 5:00 p.m. Eastern time. Telephone registration requires credit card payment; have your credit card and this form in front of you for easy reference.
FAX Transmit this form via Fax to the MRS Meeting Registration Desk (412) $367-4373$, in service 24 hours every day. Fax registration requires credit card payment.

\section{Preregistration Deadline: November 10, 1995}

NOTE: Please enter MRS I.D. No. (0...) from your mailing label if available. If not known, draw a line through code box at right.

MRS

Enter MRS I.D. No. in box.

Please fill in form completely and legibly to assure proper processing. This address is: $\square$ Business $\square$ Home $\square$ New Address $\square$ Address Correction Name

Title Last $\quad$ First/Middle Initi

Institution

Department

Street Address

P.O. Box

City.

State/Province

Zip/Postal Code

Country

Telephone $(\overline{\text { Area Coda }})$ Fax $\left(\frac{(}{\text { Area Code }}\right)$

E-Mail Address

$\square$ MRS selectively permits use of its membership list by advertisers of products which the Society deems to be of high interest to MRS members. Please check if you DO NOT wish to receive these mailings.

\section{A Meeting Preregistration}

Please check category and enter amount in payment section below.
\$275 MRS Member
\$75 MRS Student Member
$\square$ \$325 Nonmember
$\square 85$ Student Nonmember
\$105 Retired
$\square$ \$105 Unemployed
After November 10, 1995
] \$315 MRS Member
$\$ 365$ Nonmember
$\square$ \$105 Retired
$\square \$ 85$ MRS Student Member
$\$ 95$ Student Nonmember
$\square \$ 105$ Unemployed

Student registration will not be processed without proof of full-time student status. All registrations include complimentary MRS membership through December 31, 1996. Enter total here and in box below at right. TOTAL \$

Symposium interest (please check all that apply):
$\triangle A A A C \square F$
$\square \mathrm{I} \square$
$\square$ O $\square R \quad \square U$
$\square \mathrm{Y} \quad \square \mathrm{BB} \quad \square \mathrm{EE}$
$\square \mathrm{A} \quad \square \mathrm{D} \square \mathrm{G} \quad \square \mathrm{J} \quad \square \mathrm{M} \quad \square \mathrm{P} \quad \square \mathrm{S} \quad \square \mathrm{V} \quad \square \mathrm{Z} \quad \square \mathrm{CC} \quad \square \mathrm{FF}$
$\square \mathrm{B} \quad \square \mathrm{E} \quad \square \mathrm{H} \quad \square \mathrm{K} \quad \square \mathrm{N} \quad \square \mathrm{Q} \quad \square \mathrm{T} \quad \square \mathrm{W} \quad \square \mathrm{AA} \square \mathrm{DD} \square \mathrm{GG}$

Proceedings (published after this meeting)

These rates apply only to neeting attendees and MRS members. Nonmembers must contact MRS headquarters for prices.

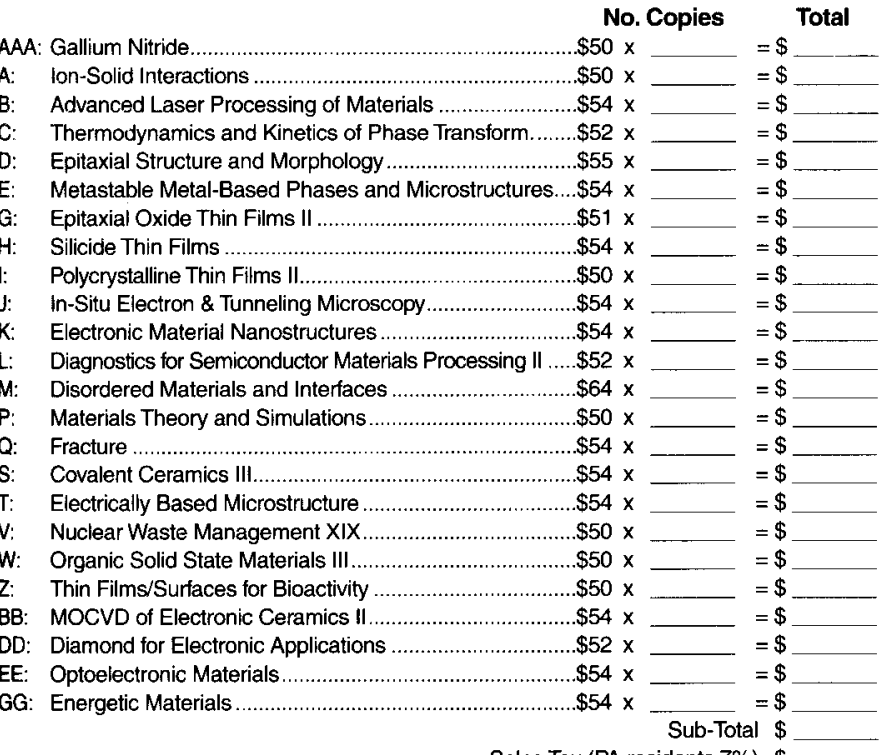

Sales Tax (PA residents 7\%)

Enter total here and in box at right. TOTAL $\$$

\section{Journal of Materials Research 1996}

Subscription at U.S. Member Rate (one per registrant) ........................ $\square \$ 75$ Subscription at Non-U.S. Member Rate (one per registrant)................. $\square \$ 88$ Optional Air Freight (non-U.S.) ............................................. $\square \$ 72$ Enter total here and in box below.

TOTAL \$

\section{Tutorials}

Tutorial fee is for course materials only. Meeting attendees may participate in all tutorial sessions at no additional charge, but will only receive the tutorial materials if preregistered for the tutorial.

There will be no refund for cancellation; however, tutorial notes will be provided.

To preregister, check each tutorial for which you want to receive materials.

FTG Epitaxial Metal Oxide Thin Films and Heterostructures......\$2

FTH Silicides for Integrated Circuits: Materials Aspects and Technological Implementation...

$\square$ FTJ In Situ Electron Microscopy: Methodology and Range of Applications.

$\square$ FTK Effects of Strain on the Electronic and Vibrational Properties of Semiconductors and Semiconductor Microstructures

$\square$ FTQ Introduction to Fracture: The Brittle/Ductile Transition and Dynamical Instabilities in Crack Propagation ..............\$25

FTW Electrical, Optical and Magnetic Properties of

Organic Solids.

FTX Federal Materials Research Programs ............................\$25

$\square$ FTc Novel Materials Research Opportunities at National Synchotron and Free Electron Laser Facilities ..................\$25

FTd Electron Emission from Diamond .................................\$25 Enter total here and in box below. TOTAL $\$$

\section{PAYMENT OPTIONS}

$\square$ Payment is enclosed. Make checks payable, in U.S. dollars, to Materials Research Society. Payment from outside the U.S. should be drawn on a correspondent U.S. bank.

Credit card payment: $\square$ Visa $\square$ MasterCard $\square$ Diners Club $\square$ AmEx Card No. Exp.

Signature

A Meeting Preregistration (from left)

B Proceedings (from left)

C Journal of Materials Research (from above)

D Tutorials (from above)

TOTAL FEES PAID

$\$$

$\$$

$\$$

$\$$

$\$$

\section{PAYMENT MUST ACCOMPANY PREREGISTRATION.}

\section{Cancellation/Refunds}

If you have already registered and find that you are unable to attend the meeting, you must notify MRS in writing of your request for a refund. Refunds will be made upon receipt of this written notice, less a $\$ 25$ service charge. If you apply a portion of this refund to member dues or any MRS publications, the service charge will be waived. MRS will not honor requests made more than one calendar month after the close of the meeting.

Tutorial Registrants: There will be no refund for cancellation; however, tutorial notes will be provided. 
MRS 1995 FALL MEETING SESSION SCHEDULE • NOVEMBER 27 - DECEMBER 1, 1995

\begin{tabular}{|c|c|c|c|c|c|c|c|c|c|c|c|c|c|c|c|c|}
\hline \multirow{2}{*}{\multicolumn{2}{|c|}{ Symposium }} & \multirow{2}{*}{ Location } & \multicolumn{3}{|c|}{ Monday, 11/27 } & \multicolumn{3}{|c|}{ Tuesday, 11/28 } & \multicolumn{3}{|c|}{ Wednesday, 11/29 } & \multicolumn{3}{|c|}{ Thursday, 11/30 } & \multicolumn{2}{|c|}{ Friday, 12/1 } \\
\hline & & & a.m. & p.m. & eve.* & a.m. & p.m. & eve.* & a.m. & p.m. & eve.* & a.m. & p.m. & eve.* & a.m. & p.m. \\
\hline AAA: & : Gallium Nitride & Republic (S) & & & \% & & & 18 & & & st & & & (9) & & \\
\hline A: & $\begin{array}{l}\text { Ion-Solid } \\
\text { Interations }\end{array}$ & $\underset{(M)}{\text { Salon } E}$ & & & 106 & & & wh & & & wo & & & w & & \\
\hline B: & $\begin{array}{l}\text { Adv. Laser } \\
\text { Process. of Matls. }\end{array}$ & $\begin{array}{c}\text { Salon } A / B \\
(M)\end{array}$ & & & 18 (8) & & & & & & s) & & & & & \\
\hline $\mathrm{C}:$ & $\begin{array}{l}\text { Thermody/Kinetics } \\
\text { of Phase Transf. }\end{array}$ & $\begin{array}{c}\text { Salon } H / 1 \\
(M)\end{array}$ & & & & & & & & & & & & 81 & & \\
\hline $\mathrm{D}:$ & $\begin{array}{l}\text { Evol. of Epitaxial } \\
\text { Structure \& Morph. }\end{array}$ & $\begin{array}{c}\text { Salon } C / D \\
(M)\end{array}$ & & & 8 & & & & & & & & & (9) & sit Ger & 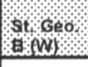 \\
\hline E: & $\begin{array}{l}\text { Metast. Metal- } \\
\text { Based Phases/ } \\
\text { Microstructure } \\
\end{array}$ & $\begin{array}{l}\text { Suffolk } \\
\text { (M) }\end{array}$ & & & wo & & & & & & & & & & & \\
\hline $\mathbf{F}:$ & $\begin{array}{l}\text { Defects in } \\
\text { High } T_{c}\end{array}$ & $\begin{array}{l}\text { Salon } G \\
(M)\end{array}$ & & & \% & & & w/ & 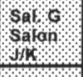 & sar. & W & & & & & \\
\hline G: & $\begin{array}{l}\text { Epitax Oxide Thin } \\
\text { Films il } \\
\text { (Sunday Tutorial) }\end{array}$ & $\begin{array}{l}\text { Salon } J / K \\
(M)\end{array}$ & & & (s) & & & (8) & sat. 6 & sarto & $10 \%$ & & & & & \\
\hline $\mathrm{H}:$ & Silicide Thin Films & $\begin{array}{c}\text { Essex East } \\
(\mathrm{M})\end{array}$ & & Tutorial & & & & 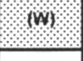 & & & & & & $w$ & & \\
\hline I: & $\begin{array}{l}\text { Polycrystalline } \\
\text { Thin Films }\end{array}$ & $\begin{array}{c}\text { Salon } F \\
(\mathrm{M})\end{array}$ & & & & & & & & & $w \%$ & & & & & \\
\hline $\mathrm{J}:$ & $\begin{array}{l}\text { In Situ Electron \& } \\
\text { Tunnel. Micros. of } \\
\text { Dynamic Proc. }\end{array}$ & $\begin{array}{l}\text { Wellesley } \\
(\mathrm{M})\end{array}$ & Tutorial & & & & & & & & & & & $w$ & & \\
\hline $\mathrm{K}:$ & $\begin{array}{l}\text { Electronic Material } \\
\text { Nanostructures }\end{array}$ & $\begin{array}{l}\text { Essex North } \\
\text { Center }(M) \\
\end{array}$ & Tutorial & & & & & w & & & & & & & & \\
\hline L: & $\begin{array}{l}\text { Semiconductor } \\
\text { Matls. Processing }\end{array}$ & $\begin{array}{l}\text { Essex West } \\
\text { M) }\end{array}$ & & & & & & w & & & & & & & & \\
\hline M: & $\begin{array}{l}\text { Disordered Matls. } \\
\text { \& Interfaces }\end{array}$ & $\begin{array}{l}\text { America } \\
\text { Center } M\end{array}$ & & & $10 \%$ & & & w: & & & w & & & & & \\
\hline $\mathrm{N}:$ & Complex Fluids & $\begin{array}{l}\text { America } \\
\text { South }(M)\end{array}$ & & Grinur: & w & & & & 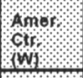 & & & & & & & \\
\hline $0:$ & $\begin{array}{l}\text { Self-Assembling } \\
\text { Matls.IStructures }\end{array}$ & $\begin{array}{c}\text { Faiffax } A \\
(S)\end{array}$ & & & & & & & & & $8 \%$ & & & & & \\
\hline$P:$ & $\begin{array}{l}\text { Matts. Theory, Sim. } \\
\text { Parallel Algorithms }\end{array}$ & $\begin{array}{c}\text { Essex South } \\
\text { M) }\end{array}$ & & & & & & & & & $m$ & & & (w) & & \\
\hline Q: $\quad$ & Fracture & $\begin{array}{l}\text { Staffordshire } \\
\text { M) }\end{array}$ & Tutorial & & & & & & 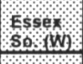 & Sossex & & & & $m$ & & \\
\hline $\mathrm{R}: \quad \underline{c}$ & $\begin{array}{l}\text { Cement-Based } \\
\text { Systems }\end{array}$ & $\begin{array}{c}\text { Essex } \\
\text { Center }(M)\end{array}$ & & & & & & & atior & & & $(5)$ w & & & & \\
\hline s: $\quad$ c & Covalent Ceramics & $\begin{array}{c}\text { Indepen- } \\
\text { dence East }\end{array}$ & & & 188 & & & & & & & & & & & \\
\hline $\mathrm{T}:$ & $\begin{array}{l}\text { Elec. Based Micro- } \\
\text { Struc. Characteriz. } \\
\end{array}$ & $\begin{array}{c}\begin{array}{c}\text { Indepen- } \\
\text { dence West }(S)\end{array} \\
\end{array}$ & & & sis & & & & & & & & & & & \\
\hline U: & $\begin{array}{l}\text { Materials Inspired } \\
\text { by Biology }\end{array}$ & $\begin{array}{c}\text { Fairfax } B \\
(\mathrm{~S})\end{array}$ & & & & & & (5) & & & & & & & & \\
\hline v: & $\begin{array}{l}\text { Nuclear Waste } \\
\text { Management }\end{array}$ & $\begin{array}{l}\text { America } \\
\text { North (M) } \\
\end{array}$ & & & & & & 18 & & & & & & & & \\
\hline W: & $\begin{array}{l}\text { Organic Solid } \\
\text { State Materials } \\
\text { (Sunday Tutorial) }\end{array}$ & $\begin{array}{l}\text { Common- } \\
\text { wealth (S) }\end{array}$ & & & m & & & & & & ss & & & & chson & Ctristim \\
\hline$x:$ & $\begin{array}{l}\text { Frontiers of Matls. } \\
\text { Research } \\
\text { (Sunday Tutorial) }\end{array}$ & $\begin{array}{l}\text { Salon } C / D \\
(M)\end{array}$ & & & & & & & & & & & & & & \\
\hline$Y: \quad$ & $\begin{array}{l}\text { Performance in } \\
\text { Polymers }\end{array}$ & $\begin{array}{c}\text { Berkeley } \\
\text { (S) }\end{array}$ & & & & & & & & & & & & & & \\
\hline Z: & $\begin{array}{l}\text { Thin Films/Surf. } \\
\text { for Bioactivity' } \\
\text { Biomed. Appl. }\end{array}$ & $\begin{array}{l}\text { Jefferson } \\
\text { (S) }\end{array}$ & & & & & & & & & & & & & & \\
\hline AA: & $\begin{array}{l}\text { Low-Cost Mfg. } \\
\text { Materials }\end{array}$ & $\begin{array}{l}\text { Regis } \\
\text { (M) }\end{array}$ & & & & & & & & & & & & & & \\
\hline BB: & $\begin{array}{l}\text { MOCVD of Elec. } \\
\text { Ceramics }\end{array}$ & $\begin{array}{c}\text { Hampton } \\
(\mathrm{S})\end{array}$ & & & & & & (s) & & & & & & & & \\
\hline $\mathrm{CC}:$ & $\begin{array}{l}\text { Spectroscopy of } \\
\text { Heterojunctions }\end{array}$ & $\begin{array}{l}\text { Yarmouth/ } \\
\text { Vineyard (M) }\end{array}$ & & & & & Tutorial & & & & & & & & & \\
\hline DD: & $\begin{array}{l}\text { Diamond for } \\
\text { Electr. Appi. }\end{array}$ & $\begin{array}{c}\begin{array}{c}\text { Constitution } \\
\text { (S) }\end{array} \\
\end{array}$ & & & & & Tutorial & 15 & & & & & & & & \\
\hline $\mathrm{EE}:$ & $\begin{array}{l}\text { Optoelectronic } \\
\text { Materials }\end{array}$ & $\begin{array}{c}\text { Gardner } \\
(S)\end{array}$ & & & & & & & & & s) & & & (1) & & \\
\hline $\mathrm{FF}:$ & $\begin{array}{l}\text { Fuilerenes, } \\
\text { Buckytubes etc. }\end{array}$ & $\begin{array}{l}\text { Indepen, } \\
\text { Centre (S) }\end{array}$ & & & & & & & & & w & & & & & \\
\hline GG: & Energetic Materials & $\begin{array}{c}\text { St. George B } \\
\text { (M) }\end{array}$ & & & & & & & & & & & & & & \\
\hline
\end{tabular}

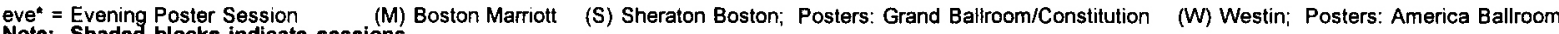




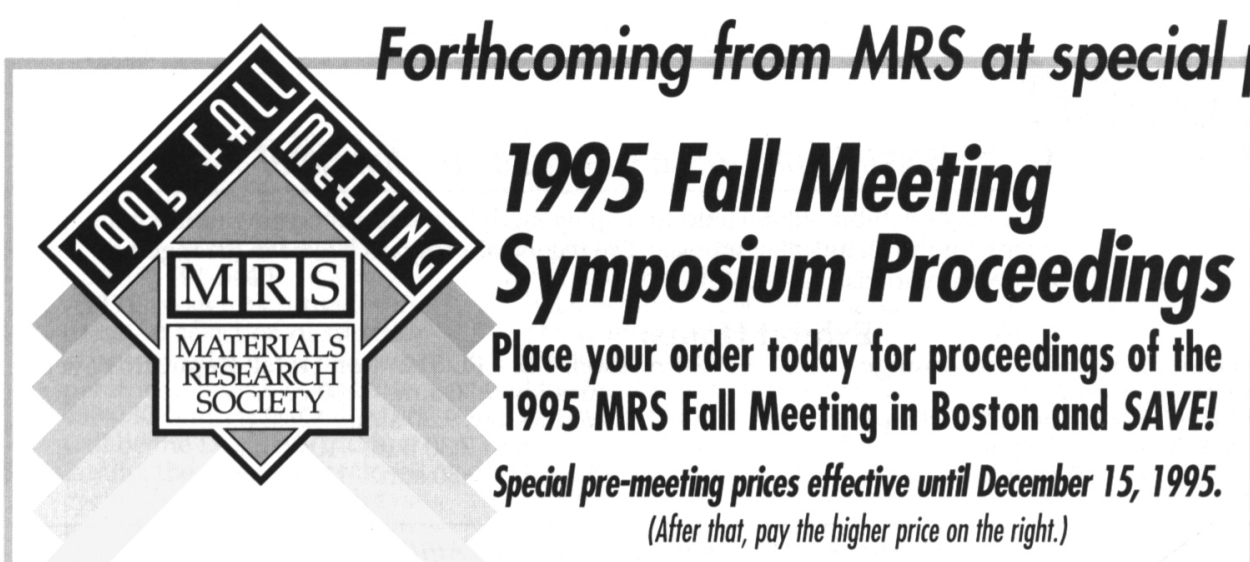

AAA: Gallium Nitride and Related Materials: The First International Symposium on Gallium Nitride and Related Materials

Editors: R.D. Dupuis, J.A. Edmond, F.A. Ponce, S. Nakamura

ISBN: 1-55899-298-7 Code: 395-B

$\begin{array}{lll}\$ 50.00 & \$ 60.00 & \text { MRS Member } \\ \$ 60.00 & \$ 65.00 & \text { U.S. List } \\ \$ 65.00 & \$ 70.00 & \text { Non-U.S. }\end{array}$

A: Ion-Solid Interactions for Materials Modification and Processing

Editors: D.B. Poker, D. Ila, Y-S. Cheng, L.R. Harriott, T.W. Sigmon ISBN: I-55899-299-5 Code: 396-B

$\begin{array}{lll}\$ 50.00 & \$ 60.00 & \text { MRS Member } \\ \$ 60.00 & \$ 65.00 & \text { U.S. List } \\ \$ 65.00 & \$ 70.00 & \text { Non-U.S. }\end{array}$

B: Advanced Laser Processing of Materials - Fundamentals and Applications

Editors: R.K. Singh, D. Norton, J. Narayan, J. Cheung, L.D. Laude ISBN: 1-55899-300-2

$\begin{array}{lll}\$ 54.00 & \$ 68.00 & \text { Mod } \\ \$ 68.00 & \$ 75.00 & \text { MRS Member } \\ \$ 75.00 & \$ 80.00 & \text { U.S. List } \\ & & \text { Non-U.S. }\end{array}$

C: Thermodynamics and Kinetics of Phase Transformations Editors: J.S. Im, B. Park, A.L. Greer, G.B. Stephenson ISBN: 1-55899-301-0

$\begin{array}{lll}\$ 52.00 & \$ 65.00 & \text { MRS Member } \\ \$ 65.00 & \$ 71.00 & \text { U.S. List } \\ \$ 71.00 & \$ 76.00 & \text { Non-U.S. }\end{array}$

D: Evolution of Epitaxial Structure and Morphology

Editors: A. Zangwill, D. Jesson, D. Chambliss, R. Clarke

ISBN: I-55899-302-9

$\begin{array}{lll}\$ 55.00 & \$ 69.00 & \text { MRS Member } \\ \$ 69.00 & \$ 76.00 & \text { U.S. List } \\ \$ 76.00 & \$ 81.00 & \text { Non-U.S. }\end{array}$

E: Metastable Metal-Based Phases and Microstructures Editors: R. Bormann, G. Mazzone, R.S. Averback, R.D. Shull, R.F. Ziolo ISBN: 1-55899-303-7

$\begin{array}{lll}\$ 54.00 & \$ 68.00 & \text { MRS Member } \\ \$ 68.00 & \$ 73.00 & \text { U.S. List } \\ \$ 73.00 & \$ 78.00 & \text { Non-U.S. }\end{array}$

G: Epitaxial Oxide Thin Films II

Editors: D.K. Fork, J.S. Speck, I. Shiosaki, R.M. Wolf

ISBN: 1-55899-304-5

Code: 40I-B

$\begin{array}{lll}\$ 51.00 & \$ 64.00 & \text { MRS Member } \\ \$ 64.00 & \$ 70.00 & \text { U.S. List } \\ \$ 70.00 & \$ 75.00 & \text { Non-U.S. }\end{array}$

H: Silicide Thin Films - Fabrication, Properties and Applications

Editors: R.T. Tung, K. Maex, P.W. Pellegrini, L.H. Allen

ISBN: 1-55899-305-3 Code: 402-B

$\begin{array}{lll}\$ 54.00 & \$ 67.00 & \text { MRS Member } \\ \$ 67.00 & \$ 73.00 & \text { U.S. List } \\ \$ 73.00 & \$ 78.00 & \text { Non-U.S. }\end{array}$

I: Polycrystalline Thin Films: Structure, Texture, Properties and Applications II

Editors: H.J. Frost, C.A. Ross, M.A. Parker, E.A. Holm

ISBN: 1-55899-306-1 Code: 403-B

$\begin{array}{lll}\$ 50.00 & \$ 60.00 & \text { Mod } \\ \$ 60.00 & \$ 65.00 & \text { U.S. List } \\ \$ 65.00 & \$ 70.00 & \text { Non-U.S. }\end{array}$

J: In Situ Electron and Tunneling Microscopy of Dynamic Processes

Editors: R. Sharma, P.L. Gai, L.J. Whitman, M. Gajdardziska-Josifovska, R. Sinclair

$\begin{array}{ccc}\text { ISBN: 1-55899-307-X } & \text { Code: 404-B } \\ \$ 54.00 & \$ 68.00 & \text { MRS Member } \\ \$ 68.00 & \$ 73.00 & \text { U.S. List } \\ \$ 73.00 & \$ 78.00 & \text { Non-U.S. }\end{array}$

K: Surface/Interface and Stress Effects in Electronic

Material Nanostructures

Editors: S.M. Prokes, K.L. Wang, R.C. Cammarata, A. Christou

ISBN: 1-55899-308-8 Code: 405-B

$\begin{array}{lll}\$ 54.00 & \$ 68.00 & \text { MRS Member } \\ \$ 68.00 & \$ 73.00 & \text { U.S. List } \\ \$ 73.00 & \$ 78.00 & \text { Non-U.S. }\end{array}$

L: Diagnostic Techniques for Semiconductor Materials Processing II

Editors: S.W. Pang, 0.J. Glembocki, F.H. Pollak, F. Celii, C.M.S. Torres ISBN: 1-55899-309-6 Code: 406-B

$\begin{array}{lll}\$ 52.00 & \$ 62.00 & \text { MRS Member } \\ \$ 62.00 & \$ 67.00 & \text { U.S. List } \\ \$ 67.00 & \$ 72.00 & \text { Non-U.S. }\end{array}$

M: Disordered Materials and Interfaces - Fractals, Structure and Dynamics

Editors: H.E. Stanley, H.Z. Cummins, D.J. Durian, D.L. Johnson

ISBN: 1-55899-310-X Code: 407-B

P: Materials Theory, Simulations, and Parallel Algorithms Editors: E. Kaxiras, J. Joannopoulos, P. Vashishta, R.K. Kalia

ISBN: 1-55899-311-8 Code: 408-B

$\begin{array}{lll}\$ 50.00 & \$ 60.00 & \text { MRS Member } \\ \$ 60.00 & \$ 65.00 & \text { U.S. List } \\ \$ 65.00 & \$ 70.00 & \text { Non-U.S. }\end{array}$

Q: Fracture - Instability Dynamics, Scaling and Ductile/Brittle Behavior

Editors: R.B. Selinger, J. Mecholsky, E.R. Fuller, Jr., A. Carlsson

ISBN: 1-55899-312-6 Code: 409-B

$\begin{array}{lll}\$ 54.00 & \$ 68.00 & \text { MRS Member } \\ \$ 68.00 & \$ 75.00 & \text { U.S. List } \\ \$ 75.00 & \$ 80.00 & \text { Non-U.S. }\end{array}$

S: Covalent Ceramics III-Science and Technology of NonOxides

Editors: G.S. Fischman, A.F. Hepp, P.N. Kumta, A.E. Kaloyeros,

J.J. Sullivan

$\begin{array}{ccc}\text { ISBN: } 1-55899-313-4 & \text { Code: 410-B } \\ \$ 54.00 & \$ 68.00 & \text { MRS Member } \\ \$ 68.00 & \$ 75.00 & \text { U.S. List } \\ \$ 75.00 & \$ 80.00 & \text { Non-U.S. }\end{array}$

T: Electrically-Based Microstructural Characterization Editors: R.A. Gerhardt, S.R. Taylor, E.J. Garboczi

ISBN: 1-55899-314-2 Code: 4II-B

$\begin{array}{lll}\$ 54.00 & \$ 68.00 & \text { Code: } 411-B \\ \$ 68.00 & \$ 75.00 & \text { MRS Member } \\ \$ 75.00 & \$ 80.00 & \text { U.S. List } \\ & & \text { Non-U.S. }\end{array}$

$\begin{array}{lll}\$ 64.00 & \$ 77.00 & \text { MRS Member } \\ \$ 77.00 & \$ 82.00 & \text { U.S. List } \\ \$ 82.00 & \$ 87.00 & \text { Non-U.S. }\end{array}$

V: Scientific Basis for Nudear Waste Management XIX

Editors: W.M. Murphy, D.A. Knecht

ISBN: 1-55899-315-0

$\begin{array}{lll}\$ 50.00 & \$ 60.00 & \text { Code: } 412-8 \\ \$ 60.00 & \$ 65.00 & \text { MRS Member } \\ \$ 65.00 & \$ 70.00 & \text { U.S. List } \\ & \text { Non-U.S. }\end{array}$

W: Electrical, Optical, and Magnetic Properties of Organic Solid State Materials III

Editors: L.R. Dalton, A. K-Y. Jen, G.E. Wnek, M.F. Rubner, C. Y.C. Lee, L.Y. Chiang

$\begin{array}{ccc}\text { ISBN: 1-55899-316-9 } & \text { Code: 413-B } \\ \$ 50.00 & \$ 59.00 & \text { MRS Member } \\ \$ 59.00 & \$ 65.00 & \text { U.S. List } \\ \$ 65.00 & \$ 69.00 & \text { Non-U.S }\end{array}$

$\$ 65.00 \quad \$ 69.00 \quad$ Non-U.S.

Z: Thin Films and Surfaces for Bioactivity and Biomedical Applications

Editors: C.M. Cotell, S.M. Gorbatkin, G. Grobe, A.E. Meyer

ISBN: 1-55899-317-7 Code: 414-B

$\begin{array}{lll}\$ 50.00 & \$ 60.00 & \text { MRS Member } \\ \$ 60.00 & \$ 65.00 & \text { U.S. List } \\ \$ 65.00 & \$ 70.00 & \text { Non-U.S. }\end{array}$

BB: Metal-Organic Chemical Vapor Deposition of Electronic Ceramics II

Editors: S.B. Desu, D.B. Beach, P.C. Van Buskirk

ISBN: 1-55899-318-5 Code: 415-B

$\begin{array}{lll}\$ 54.00 & \$ 68.00 & \text { MRS Member } \\ \$ 68.00 & \$ 75.00 & \text { U.S. List } \\ \$ 75.00 & \$ 80.00 & \text { Non-U.S. }\end{array}$

DD: Diamond for Electronic Applications

Editors: D. Dreifus, A. Collins, C. Beetz, I. Humphreys, K. Das, P. Pehrsson ISBN: 1-55899-319-3

$\begin{array}{lll}\$ 52.00 & \$ 65.00 & \text { Code: } 416-B \\ \$ 65.00 & \$ 71.00 & \text { MRS Member } \\ \$ 71.00 & \$ 76.00 & \text { U.S. List } \\ & \text { Non-U.S. }\end{array}$

EE: Optoelectronic Materials - Ordering, Composition

Modulation, and Self-Assembled Structures

Editors: E.D. Jones, A. Mascarenhas, P. Petroff, R. Bhat

ISBN: 1-55899-320-7 Code: 417-B

$\begin{array}{lll}\$ 54.00 & \$ 68.00 & \text { MRS Member } \\ \$ 68.00 & \$ 73.00 & \text { U.S. List } \\ \$ 73.00 & \$ 78.00 & \text { Non-U.S. }\end{array}$

GG: Decomposition, Combustion, and Detonation

Chemistry of Energetic Materials

Editors: T.B. Brill, T.P. Russell, W.C. Tao, R.B. Wardle

ISBN: 1-55899-321-5

\begin{tabular}{lll}
$\$ 54.00$ & $\$ 68.00$ & \multicolumn{1}{c}{ Code: $418-B$} \\
$\$ R S$ Member \\
$\$ 68.00$ & $\$ 73.00$ & U.S. List \\
$\$ 73.00$ & $\$ 78.00$ & Non-U.S.
\end{tabular}

MRS Materials Research Society M RS 9800 Mcknight Road

MATERIALS Pittsburgh, PA 15237

RESEARCH Phone: 412-367-3012

SOCIETY Fax: 412-367-4373

In Europe, Africa and the Middle East:

Clarke Associates-Europe, Ltd.

Fourth Floor The Rackhay

Queen Charlotte Street

Bristol BSI 4HJ

ENGLAND

Phone: 01179268864

Fax: 01179226437

These books are scheduled for publication by spring or early summer 1996.

Free shipping (UPS/surface only) on all prepaid orders. Air freight is additional. Standard shipping and handling charges of $\$ 3$ per book will be added to each order that is not prepaid. Contact MRS for special shipping options. 


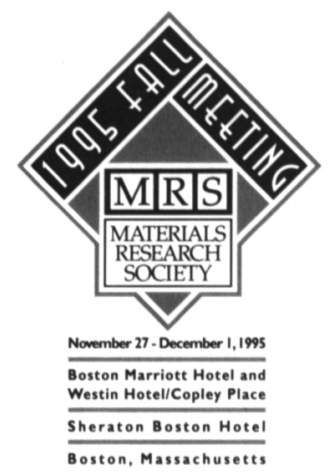

MRS Exhibit

Boston Marriott Hotel and Westin Hotel/Copley Place • Sheraton Boston Hotel

As part of the 1995 MRS Fall Meeting, a major exhibit will be held to display analytical and processing equipment and publications closely paralleling the nature of the technical symposia. The technical program has been arranged to allow meeting participants ample opportunity to visit the exhibit.

\author{
Marriott Hotel, University Hall \& Atrium Lounge Westin Hotel, 3 \& 4 Fl./Sheraton Hotel, Republic Foyer \\ Tuesday, noon - 7:00 p.m. \\ Complimentary Reception 5:00 - 7:00 p.m. \\ Wednesday, 9:30 a.m. - 5:00 p.m. \\ Thursday, $9: 30$ a.m. - 2:00 p.m.
Wednesday, 9:30 a.m. - 12:30 p.m. and $7: 30$ p.m. $-10: 00$ p.m.
Thursday, 9:30 a.m. - 12:30 p.m. \\ Tuesday, 9:30 a.m. - 5:00 p.m.
}

\title{
Partial List of 1995 Fall Exhibitors (as of September 8, 1995) \denotes Corporate Affiliate:
}

A\&N Corporation \#U960 P.O. Box 878

Inglis, FL 34449-0878

Contact: Bob Tourigny

Telephone: (800) FLANGEI

Fax: (904) 447-2322

A\&N Corporation has been a manufacturer of high vacuum components since 1965. Our product lines include: ISO-KF (QF) flanges, ISO-MF (LF) flanges, UHV (CF) flanges, ASA flanges, Vacuum couplings, Feedthroughs, and Vacuum Ball Valves. We maintain extensive inventories of all catalogued items, and routinely ship products on a same or next day basis.

\section{-ABB Extrel \#U912}

A Division of ABB Process Analytics, Inc.

575 Epsilon Drive

Pittsburgh, PA 15238-2838

Contact: Rita M. Smith

Telephone: (412) 967-5763

Fax: (412) 963-6578

ABB Extrel, a division of ABB Process Analytics, Inc., (formerly Extrel), has manufactured highquality quadrupole mass spectrometers for over 30 years. Components are combined to analyze neutrals and ions with masses up to 9,000 amu. Application areas include: surface analysis, thermal desorption and gas analysis. Real time signal processing is accomplished with the dual processor "Merlin" electronics and data system.

Academic Press, Inc. \#U106 525 B Street, Suite 1900 San Diego, CA 92101-4495 Contact: Stephanie Montell Telephone: (619) 699-6774 Fax: (619) 699-6580

With $50+$ years of experience, Academic Press continues its quest for excellence in scientific, technical publishing. Our three offices, San Diego, California, Cambridge, Massachusetts, and London, England, collectively publish 400 new book titles and 212 journals each year.

\section{Advanced Control Systems Corp. \#U602 10 Old Mine Rock Way Hingham, MA 02043 Contact: Leopold Bric Telephone: (617) 740-0223 Fax: (617) 740-4227}

ACS designs and manufactures controllers and drivers for stepping motors. Rack mounted modules include an indexer with RS232 serial control port, and drivers for both 2 and 5 phase motors, including microstepping. Board level controller/drivers include a bipolar drive capable of operating 4 motors independently.

\section{Ahlburg Technical Equipment Corp.TWELL \#A19} 6275 Atlantic Blvd.

Norcross, GA 30071

Contact: Heiko R. Ahlburg

Telephone: (404) 263-8991

Fax: (404) 449-7275

WELL diamond wire saws offer a highest degree of precision in the areas of metallography research and development and quality control. The versatility of diamond wire cutting is practically limitless. A thin diamond-coated wire of high tensile strength is wound and unwound. It has no weldment (weak point) and, therefore, can be highly preloaded. The ample coating of the $20 \mathrm{~m}$ wire guarantees a long life. Every material can be cut with the axially moving wire. The cutting surfaces are clean and parallel. Sharp corners are also guaranteed on brittle materials.

\section{AIXTRON \#S81}

1569 Barclay Blvd.

Buffalo Grove, IL 60089

Contact: Andreas Melas

Telephone: (708) 215-7335

Fax: (708) 215-7341

World leading MOCVD systems manufacturer based in Germany with a USA subsidiary and service centers in many countries. Widest possible choice of grown layers with highest throughput and uniformity of deposition. Planetary ${ }^{\circledR}$ production systems in production use by several years ahead of other competitors and for the most critical applications.

\section{AJA International \#U500} 809 Country Way

P.O. Box 246

North Scituate, MA 02060

Contact: William Hale

Telephone: (617) 545-7365

Fax: (617) 545-4105

Circular and rectangular

Magnetron Sputtering

Sources and Targets, ATC
R\&D Sputtering Systems, Microwave Power Supplies and Components, "PLANAR ECR"

Sources, Diamond Film

Equipment, Electrostatic

Chucks and LN2 Cooled

Electrodes, Vacuum

Components.

(see ad in this issue)

Alcatel Vacuum Products, Inc. \#U405

67 Sharp Street

Hingham, MA 02043

Contact: Robert D. Chapman

Telephone: (617) 331-4200

Fax: (617) 331-4230

Alcatel will display advanced

high performance, low maintenance turbomolecular pumps, including Marnetically Levitated $400 \& 1000 \mathrm{~L} / \mathrm{sec}$ hybrid pumps for standard or corrosive applications. ATH 20/40 Miniature

Hybrid, engineered for portable instruments, residual gas analysis, in-situ monitoring, ATP

Models featuring new converters.

Complete dry pumping systems.

Plus a comprehensive range of

industry acclaimed "ASM-120,

121,180 , and 181 conventional

and totally dry Leak Detectors."

Aldrich Chemical Company \#U407

P.O. Box 355

Milwaukee, WI 53201

Contact: Robert W. Marshman

Telephone: (414) 273-3850 ext. 5278

Aldrich offers more than 5,000 chemical products for scientific research, product development, and analysis in the inorganic, organometallic, and materials science fields. Materials in stock ready for immediate shipment include elemental forms, alloys, inorganics, organometallics, ligands, ACS reagents and analytical standards. Custom synthesis and bulk requests are also welcomed.

Alfa Aesar \#U608

Johnson Matthey

30 Bond Street

Ward Hill, MA 01835-8099

Telephone: (800) 343-0660

Fax: (800) 322-4757

The 1995-96 Alfa Aesar Catalog features over 12,000 products including inorganics, rare earth metals and compounds, organic and organometallics, metal alkoxides, nanosize powders, pure elements and metals available from technical grade to high-purity, analytical standards, electronic materials, precious and base metal labware, glassware and many more. Items are stocked and orders are shipped the same day. Research and production quantities quoted. ISO 9002 Certified. \#W60

2376 E. Pacifica Place

Rancho Dominguez, CA 90220

Contact: Gary Liechty

Telephone: (310) 635-2466

Toll-free: (800) 950-9347

Fax: (310) 762-6808

E-mail: AlliedHT@AOL.com Specializes in providing products for metallographic, SEM. and TEM sample preparation. Items on display will be the new TEM Wedge Polisher for mechanically thinning samples to electron transparency, diamond wafering saws, calibrated polishing equipment, precision IC cross-sectioning tools and a complete array of grinding and polishing abrasives for these applications.

\section{American Chemical Society} \#U204

1155 16th Street N.W

Washington, DC 20036

Contact: Irwin Wartell

Telephone: (202) 872-4600

Toll-free: (800) 227-5558

Fax: (202) 872-6067

E-mail: acsbooks@acs.org Displayed will be numerous periodicals including Chemistry of Materials, Macromolecules, Langmuir, the Journal of Physical Chemistry, Journal of Physical and Chemical Reference Data, Journal of Chemical and Engineering Data, and the new "Chemical Health \& Safety." Also featured will be the latest books on polymer science, industrial and applied chemistry of interest to materials researchers. Literature will also be available on ACS software and electronic products.

\section{American Institute of Physics} (AIP) \#U207

500 Sunnyside Blvd.

Woodbury, NY 11797

Contact: William Phillips

Telephone: (516) 576-2411

Fax: (516) 576-2394 
$\triangle A P D$ Cryogenics, Inc. \#A4 1833 Vultee Street

Allentown, PA 18103

Contact: Robyn Gross

Telephone: (610) 791-6700

Fax: (610) 791-0440

APD Cryogenics provides the broadest selection of superior quality products for low temperature research from 1.8 to 450.0 Kelvin. At the Fall MRS will be the Displex $(\circledR$ product family: the standard in closedcycle laboratory research systems. Featured will be the twostage, $6.5 \mathrm{~K}$ system and the unique OmniPlex ${ }^{\mathrm{TM}}$ exchange gas cryostat. APD's Heli-tran $\left.{ }^{(}\right)$ open-cycle systems will also be shown.

\section{Applied Engineering}

Technologies, Ltd. \#A21

155-B New Boston Street

Woburn, MA 01801

Contact: Glenn Driscoll

Telephone: (617) 932-3221

Fax: (617) 932-9560

E-mail: 76312.102@compuserve.com AET, Ltd. will be displaying its full line of continuous flow cryostats, as well as liquid nitrogen pour fill systems and other custom cryogenic laboratory equipment.

\section{ASTeX/Applied Science and Technology. Inc. \#U805 \\ 35 Cabot Road \\ Woburn, MA 01801 \\ Contact: Richard Myer \\ Telephone: (617) 933-5560 \\ Fax: (617) 933-0750 \\ E-mail: myer@astex.com}

ASTeX provides microwave power generators, high concentration ozone generators and systems, a range of ECR plasma sources for etching and deposition, downstream microwave plasma sources for ashing, etching and oxidation of thin films, and turnkey diamond CVD systems. ASTeX distributes PVD Products' pulsed laser deposition systems and accessories.

ATV Technology, Inc. \#U103 6 Jarvis Way

Westford, MA 01886

Contact: Jay Lincoln

Telephone: (508) 392-9234

Fax: (508) 392-9234

Programmable Process Ovens with quartz chambers up to $9^{\prime \prime}$ dia $\times 16^{\prime \prime}$ long. Computer controlled temp. profile $1100^{\circ} \mathrm{C}+2^{\circ} \mathrm{C}$, ramp up /down $100^{\circ} \mathrm{C} / \mathrm{min}$, vacuum $10^{-6} \mathrm{mbar}$ multiple programmable gas inlets. Precisely controlled hot plates $50^{\circ} \mathrm{C}$ to $450^{\circ} \mathrm{C} \pm 1^{\circ} \mathrm{C}$. Micro-Manipulator System for handling of small dies. Diamond scriber for ceramics, silicon glasses, etc. Computer controlled hot plate in a vacuum/overpressure chamber.
Balzers-Pfeiffer \#U609

8 Sagamore Park Road

Hudson, NH 03051

Contact: Craig Lazinsky

Telephone: (603) 889-6888 ext. 2392

Fax: (603) 889-8573

Balzers offers a complete range of vacuum pumps including roots and rotary pumps,

UniDry ${ }^{\mathrm{TM}}$ pump, turbomolecular and turbomolecular drag pumps; helium leak detectors; process monitors; Prisma ${ }^{\mathrm{TM}}$ gas analyzer and FullRange ${ }^{\mathrm{TM}}$ compact gauge that reads from atmosphere to UHV, with or without a separate controller. Also available are laboratory coating systems starting at under $\$ 50,000$ for $R \& D$ or small-scale production.

Barnstead/Thermolyne \#A22 2555 Kerper Blvd.

Dubuque, IA 52001

Contact: Kathy Regan

Telephone: (319) 556-2241

Fax: (319) 556-0695

Barnstead/Thermolyne

Corporation is a manufacturer of laboratory, liquid handling and water purification equipment such as furnaces including muffle furnaces, high temperature, and tube furnaces, hotplates, stirrers, mixers, incubators, sterilizers, cryogenic equipment, dri baths, heating tapes, deionization, distillation, and reverse osmosis systems and bottle top dispensers and pipettors.

Bede Scientific/North Eastern Analytical \#U712-713 17 Sherman Road

P.O. Box 25

Millis, MA 02054-0025

Contact: Joan A. Flanagan

Telephone: (508) 376-4132

Fax: (508) 376-8687

Displaying Bede Scientific QC2A, $D^{3}$ and $200 / D^{3}$ High Resolution X-Ray Diffractometer Systems. Glancing Incidence Reflectometer Systems. "RADS" Rocking Curve and "REFS" Reflectivity Simulation Software. X-Ray Generators, X-Ray Tubes and

Radiation Enclosures.

BIOSYM/Molecular

Simulations \#U702.5 9685 Scranton Road

San Diego, CA 92121-3752

Contact: Cindy Dudy

Telephone: (619) 458-9990

Fax: (619) 458-0136

E-mail: cxd@biosym.com Computer modeling is being used by expert and non-expert modelers alike to predict materials properties and design new materials with improved performance. See demonstrations and talk to our expert scientists. Learn how you can use modeling to solve your problems in polymers, catalyst research, and electronic, optical, magnetic, and high performance materials.
Blake Industries, Inc. \#U703-704

660 Jerusalem Road

Scotch Plains, NJ 07076

Contact: David G. Rognlie

Telephone: (908) 233-7240

Fax: (908) 233-1354

Blake Industries will exhibit

Blake and Huber Instruments for

thin film analysis, single crystal,

Laue apparatus, and double

crystal spectrometer for precise

lattice determinations. Standard

line of $X$-ray diffraction and syn-

chrotron accessories, including

rotary table, translation stages,

slits and goniometer heads will

be shown

Buehler, Ltd. \#U958

41 Waukegan Road

Lake Bluff, IL 60044

Contact: Fran Thompson

Telephone: (800) 283-4537

Fax: (708) 295-7929

Burleigh Instruments, Inc. \#U507

Burleigh Park, P.O. Box E

Fishers, NY 14453-0755

Contact: Patricia Payne

Telephone: (716) 924-9355

Fax: (716) 924-9072

NEW! High performance ARIS-

3500 AFM/EM accessory for surface morphology and roughness measurement. Also ambient

STM that is highly affordable as an industrial/academic research instrument and available under special pricing for education. Linear positioning stages and UHV Motors for sub-Angstrom and nanometer applications.

\section{Cambridge University Press} \#U105

40 West 20th Street

New York, NY 10011-4211

Contact: James Murphy

Telephone: (212) 924-3900

Fax: (212) 691-3239

Please stop by our booth to see these and other recent titlesBarabasi and Stanley: Fractal Concepts in Surface Growth;

Mandel and Wolf: Optical Coherence and Quantum Physics; Dugdale: The Electrical Properties of Disordered Metals; and Tsvelik Quantum Field Theory in

Condensed Matter Physics.

Cameca Instruments, Inc. \#W71 204 Spring Hill Road

Trumbull, CT 06611-1356

Contact: Florence Pindrys

Telephone: (203) 459-0623

Fax: (203) 261-5506

E-mail: p01592@psilink.com CAMECA has recently added the TAP, Tomographic Atom Probe, for 3D imaging and analysis of nanostructures, to our well established product line consisting of Magnetic Sector SIMS: IMS 6f, IMS 1270 , NanoSims 50; Time-of-Flight SIMS: TOF SIMS IV; and our newest model EPMA: SX 100. All instruments represent the highest level of performance, quality and computer integration for the serious materials researcher and production support analyst.

Ceramaseal \#U510

P.O. Box 260

Route 20

New Lebanon, NY 12125

Contact: Perry Henderson

Telephone: (518) 794-7800

Fax: (518) 794-8080

Ceramaseal offers a full range of standard ultra-high vacuum feedthroughs, connectors, thermocouples, cables, viewports, and related hardware. New products include Crystal Quartz Viewports pressure rated to 500 psi, Quartz Rod Assemblies designed for accurate hermetic transmission through ultra-high vacuum environments, and high temperature micro " $\mathrm{D}$ " series connectors 9 pin through 50 pin.

CHA Industries \#U914

4201 Business Center Drive Fremont, CA 94538-6357

Contact: Shannon Pimentelli

Telephone: (510) 683-8554 CHA Industries is Vertically Integrated, with over 50 years of experience as a manufacturer and OEM supplier of standard and custom High Vacuum Systems. Manufacturers of Semiconductor, XTAL, LEDS, Optics, Automotive, and Aerospace devices use various versions of CHA's Sputtering, Evaporation, and Ion Beam Systems. CHA manufactures Roll Coaters, Box Coaters clean-room compatible, $R \& D$, and Production systems (and components) for Medium- and High-Vacuum applications.

Chapman \& Hall \#U206 115 th Avenue

New York, NY 10003

Contact: Steve Hawe

Telephone: (212) 780-6239

Fax: (212) 260-1730

E-mail: hawe@chaphall.com Chapman \& Hall, publisher of the Journal of Materials Science, will demonstrate the online version of the journal. They will 
Commonwealth Scientific Corporation \#U804

500 Pendleton Street

Alexandria, VA 22314

Contact: David Day

Telephone: (703) 548-0800

Fax: (703) 548-7405

Commonwealth Scientific Corporation (CSC) is the leader in ion beam technology, manufacturing a complete line of products for dry etching and thin film deposition processes. $\mathrm{CSC}$ will be displaying their complete line of ion sources ranging from $3 \mathrm{~cm}$ to $38 \mathrm{~cm}$ in diameter. New products include the $1 \mathrm{~m}$ gridless linear end-Hall source and the Carbon Cathodic Arc Source.

The Cooke Corporation \#U966 600 Main Street

Tonawanda, NY 14150-0888

Contact: Barry Brandon

Telephone: (716) 833-8274

Fax: (716) 836-2927

Two examples of optical direct extensometers will be on exhibit. The models' $200 X$ high resolution, high strain rate system and UDM 5000 long range CCD extensometer are capable of direct strain and compression measurements of specimens over very long periods of time (creep testing) and over a wide range of testing temperatures (up to $3000^{\circ} \mathrm{C}$ ).

Cornell Theory Center \#U102

528 Frank H.T. Rhodes Hall Ithaca, NY 14850-3801

Contact: Paul RedFern

Telephone: (607) 254-8609

Fax: (607) 254-8888

E-mail: red@tc.cornell.edu

CTC provides industry parallel computing and modeling services designed to solve materials science and engineering problems. Current clients include Abbott Labs, Biosym, Corning, Merck, SAIC, and Xerox. CTC welcomes R\&D outsourcing inquiries from large companies and small. Current projects include new materials discovery, rational drug design, and modeling manufacturing processes.

\section{Cressington Scientific}

Instruments, Inc. \#W68

508 Thomson Park Drive

Cranberry Twp., PA 16066-6425

Contact: Alan Berginc

Telephone: (412) 772-0220

Fax: (412) 772-0219

Cressington designs and manufactures a wide range of thin film vacuum coating equipment for electron microscope specimen preparation. We specialize in modern, small-footprint benchtop coating systems that accurately and repetitively deposit carbon as well as a high resolution sputter coating system for FESEM.
Cryomech, Inc. \#U604

110 Falso Drive

Syracuse, NY 13211

Contact: Peter E. Gifford

Telephone: (315) 455-2555

Fax: (315) 455-2544

Cymer Laser Technologies \#U305

16275 Technology Drive

San Diego, CA 92127-1815

Contact: Christopher Rowan

Telephone: (619) 487-2442

Fax: (619) 487-2441

E-mail: crowan@cymer.cts.com

Cymer Laser Technologies manufactures excimer lasers for industrial use. Cymer has two lines of products, a spectrally narrowed and wavelength stabilized krypton fluoride excimer laser, to be used as the photolithography stepper's illuminator source, and the high power $\mathrm{KrF}$ excimer laser for use in micromachining, surface modification and annealing.

DCA Instruments \#U950

400 W. Cummings Park, Suite 3900

Woburn, MA 01801

Contact: Jari Vanhatalo

Telephone: (617) 937-6550

Fax: (617) 935-2405

Denton Vacuum, Inc. \#U404

1259 North Church Street

Moorestown, NJ 08057

Contact: James L. Campbell

Telephone: (609) 439-9100

Fax: (609) 439-9111

Denton Vacuum, Inc. (DVI), established for over 30 years, is one of America's premier manufacturers of research and production thin film deposition systems. DVI currently offers a wide variety of standard systems to meet applications ranging from the preparation of samples for electron microscopy to thin film research requiring computer controlled, multi-source thin film sputtering systems and electron beam evaporation systems. Representatives will be available at the Fall Meeting to discuss your specific application and to suggest solutions to your equipment requirements.

Digital Instruments \#U817-818

520 E. Montecito Street

Santa Barbara, CA 93103

Contact: Terry Mehr

Telephone: (805) 899-3380

Fax: (805) 899-3392

E-mail: info@di.com

World Wide Web:

http://www.di.com/

Digital Instruments, the world

leader in Scanning Probe

Microscopy (SPM), will be

exhibiting its NanoScope $\mathbb{R}$

SPMs including the Multi-

Mode ${ }^{\mathrm{TM}}$ Atomic Force Micro-

scope (AFM), the world's best

selling and highest resolution

SPM. Featured at MRS this

year is the Dimension ${ }^{\mathrm{TM}} 3000$

SPM/AFM (Atomic Force

Microscope), offering the com-

plete range of SPM techniques for small or large samples, including topography, magnetic force, lateral force, electrochemistry, TappingMode ${ }^{\mathrm{TM}}$, force modulation and others. (see ad in this issue)

\section{Duniway Stockroom Corp.} \#U957

1305 Space Park Way

Mountain View, CA 94043

Contact: Ralph R. Duniway

Telephone: (415) 969-8811

Fax: (415) 965-0764

New replacement parts for ion pumps, leak detectors, and vacuum systems; including 12 point bolts, copper gaskets, TC gauges, ion gauges, oil for diffusion pumps, etc. Surplus vacuum equipment for sale, rebuilt to original performance. 44 page catalog, including prices, shows new and surplus equipment.

\section{Edwards High Vacuum}

International \#U409

301 Ballardvale Street

Wilmington, MA 01887

Contact: Lisa Doody

Telephone: (800) 848-9800

Fax: (508) 657-6546

Edwards High Vacuum

International, a division of the

BOC Group, is a world leading manufacturer of vacuum components and systems. Some of Edwards' products include the RV range of rotary vacuum pumps, EXP pumping units including turbo pump, controller and backing pump, active vacuum gauges, and the Barocel $®$ Capacitance Manometers. These products will be on display at the 1995 MRS Show.

Elsevier Science \#U307-310 655 Avenue of the Americas

New York, NY 10010

Contact: Traci Taylor

Telephone: (212) 633-3766

Fax: (212) 633-3764

(see ad in this issue)

EMCORE Corporation \#U913 394 Elizabeth Avenue

Somerset, NJ 08873

Contact: Mark McKee

Telephone: (908) 271-9090

Fax: (908) 271-9686

E-mail: MARK@EMCORE.com EMCORE Corporation

manufacturer of TurboDisc

Deposition systems producing highest quality thin film compound and silicon semiconductor wafers with uniformity of thickness, doping and composition, and interface abruptness required for modern electronic, microwave and optoelectronic devices. EMCORE's TurboDisc systems are further distinguished by low cost of ownership, reliability (high MTBF), throughput, capacity and process conditions and utilize major deposition technologies including MOCVD, ALE, and CVD depending on applications requirements.

$\triangle$ EPI MBE Products Group \#U100 1290 Hammond Road

St. Paul, MN 55110

Contact: Dave Reame

Telephone: (612) 653-0488

Fax: (612) 653-0725

E-mail: daver@epimbe.com EPI is North America's leading manufacturer of MBE equipment and one of the largest in the world. EPI has set the standard for excellence and has been the international leader in quality and innovation in this field since 1986. EPI provides materials-specific effusion cells and MBE systems for use with GaAs/AIGaAs, silicon, nitrides, antimonides, phosphides, and II-VI materials. In addition, EPI manufactures a Flat Panel Probe Tool for testing Field Emission Displays.

\section{E S Microware \#U408}

\section{Wade Court}

Hamilton, OH 45013

Contact: Scott McCormick

Telephone: (513) 738-4773

Fax: (513) 738-4407

E-mail: ESMicro@IAC.NET E S Microware develops and markets materials-related software for Windows and Macintosh platforms. Products include TAPP, a database of thermodynamic and physical properties of solids, liquids and gases, ChemSage, for calculation of complex thermodynamic phase equilibria, and Mat.DB, a database of properties of engineering materials.

Evans East \#U705

666 Plainsboro Road, Suite 1236

Plainsboro, NJ 08536

Contact: Rennie Harrington

Telephone: (609) 799-1904

Fax: (609) 799-8691

Analytical services performed using SIMS, XPS (ESCA), TOF- 
$\$$ E.A. Fischione Instruments, Inc. \#U710

9003 Corporate Circle

Export, PA 15632

Contact: Paul E. Fischione

Telephone: (412) 325-5444

Fax: (412) 325-5443

E.A. Fischione Instruments, Inc features a complete line of TEM Specimen Preparation devices including the Model 3000 Ion Mill, the Model 330 Ultrasonic Disk Cutter, the Model 2000 Specimen Prep System (dimple grinder), and the Automatic Twin-Jet Electropolisher. New product introductions include the Model 1400 Plasma Cleaner for the elimination of contamination from both TEM specimens and TEM Specimen Holders. Also displayed are SEM and TEM Specimen Holders including the CryoPrep Station/TEM Holder.

Fisons Instruments \#U905 55 Cherry Hill Drive

Beverly, MA 01915

Contact: Phil Sullivan

Telephone: (508) 524-1000

Fax: (508) 524-1100

E-mail: PHILIPS587@aol.com Fisons Instruments Surface Systems is the world's largest supplier of surface analysis equipment, molecular beam epitaxy systems and dedicated scanning transmission electron microscopes.

\section{Gatan, Inc. \#U701 \\ 6678 Owens Drive}

Pleasanton, CA 94588-3334

Contact: Paul V. Miller

(Eastern Sales), Sheri Kurland (Western Sales)

Telephone: (510) 463-0200

Fax: (510) 463-0204

Gatan is a technology leader in the manufacture of instrumentation for TEM, STEM and SEM. Products include a complete selection of side-entry TEM specimen holders, TEM specimen preparation equipment, TV-rate and slow-scan CCD cameras for TEM, multi-signal digital imaging system for SEM \& STEM, parallel detection electron energy loss spectrometer, and energy filtered imaging system.

Gelest \#U962

612 William Leigh Drive

Tullytown, PA 19007

Contact: Barry Arkles

Telephone: (215) 547-1015

Fax: (215) 547-2484

\section{-Gordon and Breach Publishers Harwood Academic Publishers \#U311}

820 Town Center Drive

Langhorne, PA 19047

Contact: Michelle McKenna

Telephone: (215) 750-2642 ext. 158

Fax: (215) 750-6343

Gordon and Breach/Harwood

Academic publishes books and

journals of interest to scientists

and engineers in condensed matter physics and materials science, including nanotechnology, optics and lasers, surface physics, nonlinear dynamics, and superconductivity.

\section{Harris Diamond Corporation} \#U302

100 Stierli Court, Suite 106

Mt. Arlington, NJ 07856

Contact: David Slaperud

Telephone: (201) 770-1420

Fax: (201) 770-1549

Harris Diamond Corporation is a proud supplier of Drukker diamond ultramicrotome knives manufactured by Drukker International BV, The Netherlands. Advantages include superb wetting behavior, guaranteed compressionfree sections of soft biological tissue, and an immediate exchange program. Other Advanced Diamond Products include natural and CVD diamond windows and thermal management substrates.

Heraeus Amersil, Inc. \#U911 3473 Satellite Blvd.

Duluth, GA 30136-5831

Contact: Howard Young

Telephone: (408) 281-3529

Fax: (408) 225-3830

Heraeus Amersil, Inc. is the largest fabricator of quartz glass products in the United States. It is a wholly owned subsidiary of Heraeus Quarzglas, $\mathrm{GmbH}$; the world's largest producer of high-purity quartz materials for semiconductor and optical applications.

\section{High Voltage Engineering} Europa B.V. \#U700

P.O. Box 99

3800 AB Amersfoort

The Netherlands

Contact: Henri van Oosterhout

Telephone: (31) 33-619741

Fax: (31) 33-615291

Ion accelerator systems for science and industry; Ion Implantation, Ion Beam Analysis by RBS, PIXE, NRA,

ERD, MEIS, and AMS

(see ad in this issue)

Hitachi Scientific Instruments \#U201-203

755 Ravendale Drive

Mountain View, CA 94043

Contact: Robert Gordon

Telephone: (415) 969-1100

Fax: (415) 961-0368

Hitachi, a world leader in electron microscope advancements and proven technologies, represents our full line of exceptional scanning, transmission and field emission electron microscopes by giving demonstrations of our Model S-4500. The Model S-4500 FE-SEM (with inlens SE detector) gives resolution of $40 \AA$ guaranteed at $1 \mathrm{KV}$, and $15 \AA$ guaranteed at $15 \mathrm{KV}$. This detector yields ultimate surface detail not seen with a standard SE detector. (see ad in this issue)
Huntington Mechanical Laboratories \#A14

1040 L'Avenida

Mountain View, CA 94043

Contact: Ken Bertken

Telephone: (415) 964-3323

Fax: (415) 964-6153

The industry's largest selection of vacuum valves, flanges, fit-

tings, and feedthroughs is avail-

able when you need it at

Huntington. Also available are

a wide assortment of roughing

components including flexible

hoses, traps, thermocouple and

ionization gauge tubes, sorption

and jet roughing pumps.

Standard, custom or modified

UHV positioning and motion devices can be provided to meet your special needs. Stainless steel custom chambers, tees and crosses are supported by a quarter of a century of experience in vacuum chamber design and fabrication at Huntington. (see ad in this issue)

IBM Analytical Services \#A8

1580 Route 52

Hopewell Junction, NY 12533-6531

Contact: David Fouts

Telephone: (914) 892-2450

Fax: (914) 892-2003

E-mail: dpfouts@vnet.ibm.com

Offers a broad range of capabilities from failure analysis to chemical and electrical characterization, all performed by a highly experienced staff of experts in their specific fields.

We offer high-quality work performed on state-of-the-art equipment in a timely and cost-competitive environment.

\section{Implant Sciences Corporation} \#U101

107 Audubon Road,

\#5 Corporate Place

Wakefield MA 01880

Contact: Robert Hayward

Telephone: (617) 246-0700

Fax: (617) 246-1167

Ion implantation services with over 60 species available, including noble metals and rare earths. Heated implants and cryogenic implants done in research applications. Profile Code $^{\mathrm{TM}}$ software on display for accurate simulation of ion implantation. Pin-on-disk

friction and wear testing equipment also on display.

Inel, Inc.- \#U104

P.O. Box 147

Stratham, NH 03885

Contact: Greg Stollstorff

Telephone: (603) 778-9161

Fax: (603) 778-9171

Inel specializes in manufacturing Position Sensitive Detectors (PSD), both curved and linear, for many diffraction applications including powders, polymers dynamics, in situ, on-line, thin film grazing angle, reflectometry and small angle scattering Complete PSD diffractometer systems are available, or enhance performance of existing goniometers by retrofitting a PSD.

Innovative Technology, Inc. \#U903

2 New Pasture Road

Newburyport, MA 01950

Contact: Renneth J. O'Brien

Telephone: (508) 462-4415

Fax: (508) 462-3338

Innovative Technology, Inc. will exhibit our new position sensitive detector and multichannel analyzer package. We will also display a new small angle $X$-ray camera.

Inorgtech, Ltd. \#U965

25 James Carter Road

Mildenhall

Suffolk IP28 7DE

United Kingdom

Contact: Tim Leedham

Telephone: (44) 1638-714423

Fax: (44) 1638-718259

Manufacturer of inorganic

chemicals, especially precursors

for deposition of oxide and nitride thin films by MOCVD and sol-gel. Products include tantalum ethoxide, tin tertiary butoxide, titanium dimethylamide and zirconium tertiary butoxide. Available from stock in gram to kilo quantities. Custom synthesis service.

$\$$ Institute for Scientific Information (ISI) \#U208 3501 Market Street

Philadelphia, PA 19104

Contact: Frank Spiecker

Telephone: (215) 386-0100

Fax: (215) 386-6362

E-mail: spiecker@isinet.com A secondary publisher of scientific, technical and medical information, ISI products are unique in that they allow one to search on the cited references of an article. Stop by to see a Windows version of Current Contents ${ }^{\circledR}$ and learn more about the Materials Science 
Instron Corporation \#U814 100 Royall Street

Canton, MA 02021

Contact: Kathy Hendry

Instron will be showing its latest

Fast Track software and 8500 plus

fatigue testing system and the

UMIS 2000 NANO-SCALE test-

ing system. Materials test inno-

vations for both static and dyna-

mic testing will be on display.

\section{Instruments SA, Inc./ \\ lobin-Yvon-Spex \#U908 \\ 3880 Park Avenue}

Edison, NJ 08820

Contact: Victor Yakovkv, Fran Adar

Telephone: (908) 494-8660

Fax: (908) 549-2571

Instruments SA, Inc. is committed to serving our customers with superior products and technical support for optical spectroscopy. ISA offers many versatile research and low cost Raman spectrometers as well as scanning and imaging spectrometer systems, fluorescence systems, ellipsometry, and RDS systems, photo diode array (PDA) and charge coupled device $(\mathrm{CCD})$ detectors.

Ion Tech, Inc. \#U501 2330 East Prospect

Fort Collins, CO 80525

Contact: Gerald Isaacson

Telephone: (303) 221-1807

Fax: (303) 493-1439

Ion Tech, Inc. is the industry leader for providing superior ion beam equipment. The product line ranges from linear and round DC sources to filamentless RF sources, power supplies and complete systems. This equipment can be used for etching, precleaning and the production of sophisticated thin films such as diamondlike carbon (DLC), complex alloys and high precision optical films.

Janis Research Company, Inc. \#U803

\section{Jewel Drive}

Wilmington, MA 01887-0696

Contact: Munir Jirmanus

Telephone: (508) 657-8750

Fax: (508) 658-0349

E-mail:74211.1613@compuserve.com Janis combines over 30 years of experience with extensive engineering capabilities to provide cryogenic systems for all research applications. Janis offers closed cycle refrigerators, continuous flow and variable temperature cryostats, superconducting magnet systems, detector cooling dewars, dilution refrigerators, Helium-3 cryostats including top loading, and custom designs to meet any specific requirement.

\section{IICPDS-ICDD \#A15}

Newtown Square, PA 19073

Contact: Mario Fornoff

Telephone: (610) 325-9810

Fax: (610) 325-9823

E-mail: FORNOFF@ICDD.COM

The JCPDS International Centre for Diffraction Data maintains and distributes the Powder Diffraction File database for use in materials characterization through $X$-ray analysis. The database contains approximately 62,000 numeric diffraction patterns of crystalline phases. Specialized products include Forensics, Minerals, and Metals \& Alloys subfiles as well as various educational publications.

\section{IIEOL USA, Inc. \#A7 \\ 11 Dearborn Road}

Peabody, MA 01960

Contact: Michael Kersker

Telephone: (508) 535-5900

Fax: (508) 536-2205

E-mail: Kersker@jeol.com JEOL has always relied on sound technology, steady service and support, and quality manufacturing to meet the scientific demands of the materials science community. Today, JEOL remains committed to providing the same reliable, high performance SEMs and TEMs to challenge the needs of applied and basic materials research in the future.

\section{Iohnsen Ultravac \#A12}

3470 Mainway

Burlington, ON L7M 1A8

Canada

Contact: Hans De Jong

Telephone: (905) 335-7022

Toll-free: (800) 268-4980

Fax: (905) 335-3506

E-mail: JUVINFO@

NETACCESS.ON.CA

JUV manipulators are available with 6-axis of rotation, heating/cooling stages, and the widest range in $X Y Z$ motions. Of particular interest is the new 4100 series featuring a payload capacity of 250 kilos. Our uhv systems include ECR, Evaporation, Ion Sputtering, LASER Ablation, MBE, RF/DC Sputtering and surface analysis.

\section{k-Space Associates, Inc. \#U910}

2231 Stone Road

Ann Arbor, MI 48105

Contact: Darryl Barlett

Telephone: (313) 763-8747

Fax: (313) 763-8747

E-mail: kspace@dial.cic.net

The KSA 300 family of CCD-

based imaging and analysis systems are for the scientist who wants to exploit the full power of RHEED and LEED. Computer-controlled, user-selectable on-chip time integration, combined with advanced data extraction, storage, fitting, and analysis techniques, allow users to quantitatively study, monitor, and control thin film deposition processes.

Keithley Instruments \#U909 28775 Aurora Road

Solon, OH 44139

Contact: Kristin Rice

Telephone: (216) 498-2481

Fax: (216) 248-6168
Kimball Physics, Inc. \#U504 311 Kimball Hill Road

Wilton, NH 03086

Contact: Faye Bigarel

Telephone: (603) 878-1616

Fax: (603) 878-3700

E-mail: faye@Kimphys.com UHV Electron Sources, Ion Sources, Components: $5 \mathrm{eV}$ to $100 \mathrm{keV}, 10^{-15} \mathrm{~A}$ to $1 \mathrm{~A} ; \mu \mathrm{m}$ to m Diameters. Applications: Surface Physics, RHEED, ESD, IPES, MBE, Heating, Space Physics, Semiconductor Processing, Microscopy, etc.

New UHV Components available include innovative MultiCF ${ }^{\mathrm{TM}}$ Fittings for making small UHV Chambers. Also available: Cathodes, Faraday Cups

Phosphor Screens, eV Parts.

Kluwer Academic Publishers \#U306

101 Philip Drive

Norwell, MA 02061

Contact: Annie Rollins

Telephone: (617) 871-6600

Fax: (617) 871-6528

E-mail: Kluwer@world.std.com Kluwer Academic Publishers will have on display the latest scientific books and journals in the area of materials science including ceramics, optics, chemical and structural properties research. Visit our booth and pick up a sample copy of

Kluwer's new materials science journals: Adsorption; The Journal of Porous Materials, Advanced Performance Materials; Applied Composite Materials; Interface Science and The Journal of Sol-Gel Science.

Kratos Analytical, Inc. \#W65

535 East Crescent Avenue

Ramsey, NJ 07446

Contact: David Surman

Telephone: (201) 825-7500

Fax: (201) 825-8659

E-mail: dsurman@kratos.com Kratos Analytical is a leading supplier of surface analysis instrumentation, with an emphasis on XPS, AES/SAM SIMS and ISS. The instrumentation is designed with an emphasis on materials characterization in areas such as polymers, ceramics and electronics, and is suitable for both quality control and $R \& D$ applications.

\section{Lake Shore Cryotronics, Inc. \#U706-707}

64 East Walnut Street

Westerville, $\mathrm{OH} 43081$

Contact: Michael Swartz

Telephone: (614) 891-2243

Fax: (614) 891-1392

E-mail: lsci-sales@bronze.coil.com

-Lambda Physik, Inc. \#U954

289 Great Road

Acton, MA 01720

Contact: Gerard Zaal

Telephone: (508) 263-1100

Fax: (508) 263-4296

Lambda Physik, the world

leader in excimer and pulsed

dye laser technology, has recently introduced the

COMPex series excimer laser with state-of-the-art efficiency and ease of use as well as a new FAMP Femtosecond UV-visibleNIR laser for all ultrafast applications. Our complete line of excimer and pulsed dye lasers are ideal for all high energy pulsed laser applications, such as PLD, etc.

Kurt I. Lesker Company \#U955

1515 Worthington Avenue

Clairton, PA 15025-2700

Contact: Michael McKeown

Telephone: (412) 233-4200

Toll-free: (800) 245-1656

Fax: (412) 233-4275

E-mail: 73377.1045@

compuserve.com

We are exclusive North

American agents for Vacuum

Generators, displaying their economical high precision $X Y Z$ manipulators, unique UHV sample distribution center and their surface science components for XPS, AES, UPS, LEED, RHEED. We will show our manufacturing capability for high vacuum and UHV custom chambers together with flanges, hardware, electrical and rotary feedthroughs.

Leybold Inficon, Inc. \#U902

Two Technology Place

East Syracuse, NY 13057

Contact: Lou Landman

Telephone: (914) 279-4105

Fax: (914) 279-2889

Leybold Inficon's advanced instrumentation techniques combine unique sensing technology with specialized electronics. The company's expertise includes quadrupole-based mass spectrometers and partial pressure controllers for 
staff. Precise measurement, cutting, and fabrication of magnets and assemblies are accom-

plished with state-of-the-art

in-house facilities.

(see ad in this issue)

Maxtek, Inc. \#W66

2908 Oregon Court, G-3

Torrance, CA 90506-2683

Contact: Ferne Hildebrand

Telephone: (310) 320-6604

Fax: (310) 320-6609

Maxtek manufactures two product lines: one for Thin Film Deposition, the other for liquid applications. Our Deposition Controllers combine Windowsbased PC software with dedicated hardware providing the most powerful and easy to use deposition control system anywhere. Plating monitors are applicable for electroless and electrolytic processes. Both product lines utilize QCM technology.

\section{Braun, Inc. \#W67}

2 Centennial Drive Unit $4 \mathrm{~F}$ Peabody, MA 01960

Contact: Rodney F. Denman

Telephone: (508) 531-6217

Fax: (508) 531-7245

M. Braun, Inc. will exhibit its PC-based Position Sensitive XRay Detector System, the most advanced in the industry. The platinum wire counter can perform stationary and independent/simultaneous small and wide angle measurements (with Kratky Collimator and 2 PSD's), and fast powder diffraction. Information available on our full line of Inert Atmosphere Glove Boxes and Attachments.

McPherson, Inc. \#S82

530 Main Street

Acton, MA 01720

Contact: Christl M. Schoeffel

Telephone: (508) 263-7733

Fax: (508) 263-1458

E-mail:72234.2257@

compuserve.com

McPherson designs and manufactures monochromators/spectrometers/spectrographs and turn-key spectroscopic systems for the XUV, VUV, UV, VIS, and IR range. Accessories such as light sources, detectors, and components for sample handling as well as accessories for systems in X-ray lithography and synchrotron radiation are standard catalog items.

\section{Vacuum Products Corporation \#U702 23842 Cabot Blvd}

Hayward, CA 94545

Contact: Mike Weiss, Phil Crane Telephone: (510) 887-6100

Fax: (510) 887-0626

MDC manufactures a complete line of UHV stainless steel vacuum components including flanges and fittings, valves, chambers, electron beam sources, electrical feedthroughs, motion feedthroughs, roughing components, instrumentation and other accessories. A full technical staff is maintained to assist with technical questions and the design and manufacture of custom components and chambers.

(see ad in this issue)

Media Cybernetics \#U200

8484 Georgia Avenue

Silver Spring, MD 20910

Contact: Shahira Hoffman

Telephone: (301) 495-3305

Fax: (301) 495-5964

Media Cybernetics will demonstrate Image-Pro Plus and is introducing Materials-Pro Analyzer. Image-Pro is an image analysis software package which provides counting sizing, statistical and image enhancement operations with an intuitive and consisten interface designed to produce results quickly. Materials-Pro Analyzer is a set of powerful applications with a focused design on materials analysis and reporting of grains, porosity, inclusions, particles, phase discrimination, and nodularity.

Melles Griot \#A17

1770 Kettering Street

Irvine, CA 92714

Telephone: (714) 261-5600

Toll-free: (800) 835-2626

Fax: (714) 261-7589

Melles Griot, worldwide manufacturers of Optics, Lasers, Opto-Mechanics and Instruments, will be demonstrating a variety of optical assemblies and components, optical instrumentation, colored HeNe lasers, diode laser assemblies and positioning equipment.

Representatives will be ready to assist you in a wide variety of photonic applications.

Micro Instrument Company \#A18

2250 Micro Place

Escondido, CA 92029

Contact: Curt Haas,

Charlie Marks, or Ted Nation

Telephone: (619) 746-2010

Fax: (619) 746-0433

Micro Instrument Company provides turn-key system solutions for integrated circuit reliability testing including Time Dependent Dielectric Break down (including v-ramp), Electromigration (including Temperature Coefficient of Resistance, Continuous Current, and SWEAT), and Hot Carried Induced Degradation. Systems provide testing capability for packaged products and wafers. Micro Instrument Company solutions feature selfcalibration, temperature and measurement accuracy, and full system specifications. Robust control and analysis software provide integrated solutions for the quality researcher. Visit the Micro Instrument Company booth for a hands-on demonstration of the Model PE9020, a small-lot test system for electro- migration and time dependent dielectric breakdown.

Micro Photonics, Inc. \#W61

Surface Test Division

P.O. Box 3129

Allentown, PA 18106

Contact: George Ferrio

Telephone: (610) 366-7103

Fax: (610) 366-7105

E-mail: surftest@aol.com

Surface Test, a division of Micro

Photonics, offers instrumentation for measuring mechanical properties of thin films and bulk materials including hardness, friction, adhesion, scratch resistance, wear resistance and surface roughness. Also offered are ellipsometers, laser interferometers and optical emission spectrometers for in-situ monitoring and process control.

Microcal Software, Inc. \#U959

One Roundhouse Plaza

Northampton, MA 01060

Contact: Claudine Witmer

Telephone: (413) 586-2013

Fax: (413) 585-0126

Microcal Software, Inc. offers

Origin, a technical graphics,

data analysis, and data acquisi-

tion software in Windows.

Origin is widely recognized as the most powerful, fast, and easy-to-use software of its kind. Features built-in and userdefined statistical, mathematical, and curve-fitting functions, and an open-ended architecture supporting numerous add-on modules.

Mikron Instrument Company, Inc. \#A20

445 West Main Street

Wyckoff, NJ 07481

Contact: Mel Jenkins

Telephone: (201) 891-7330

Fax: (201) 891-1205

Mikron's M9000 Imaging

Pyrometer uses a unique

patented solid state detector array camera to operate in the near infrared part of the spectrum, measuring from 500-

$300^{\circ} \mathrm{C}$. Performance is enhanced by high resolution with $640 \times 480$ pixel image, 30 frames per second response and elimination of image blooming and light scattering.

MKS Instruments, Inc. \#U708-709

Six Shattuck Road

Andover, MA 01810

Contact: Applications

Engineering Dept.

Telephone: (800) 227-8766

Fax: (508) 975-0093

MKS will exhibit its full line of vacuum/pressure/flow instrumentation, including mass flow meters and controllers, vacuum gauges, pressure transducers, pressure control systems, HPS vacuum valves and hardware, and NGS mass spectrometers and primary and secondary calibration systems. Direct Liquid Chemical Injection systems will also be featured.

MMR Technologies \#U509

1400 N. Shoreline Blvd., Suite A-5 Mountain View, CA 94043-1346

Contact: Robert L. Paugh

Telephone: (415) 962-9620

Fax: (415) 962-9647

MMR Technologies manufactures Temperature Controlled Systems-Cryogenic Cooling Systems and Wide Temperature Range Thermal Stages-which find application in Materials Research. These systems are used in Physics and Chemistry Research Applications over the temperature range of $10 \mathrm{~K}$ to $580 \mathrm{~K}$. They are also used in the Cooling of Electronic Devices, Laser Diodes, and Thermal Imaging Devices and in the Characterization of the performance and properties of such

Devices as a function of

Temperature.

MR Semicon, Inc. \#A9

P.O. Box D-1900

Pomona, NY 10970-0499

Contact: Patricia Ferguson

Telephone: (914) 362-6771

Toll-free: (800) 832-1518

Fax: (914) 362-7723

MR Semicon, Inc. will be showing a full range of bench top sample preparation equipment, featuring the NEW LabOne chemically resistant polishing system and LabTrim, the inexpensive cutoff saw. Also manufactures, markets and refurbishes a complete range of crystal manufacturing equipment for production, research and proof of concept; Czochralski, top seeded flux, Bridgman \& float zone.

n \& k Technology Inc. \#U606 3150 De La Cruz Blvd., Suite 105 Santa Clara, CA 95054 
size ranges, phases, dispersions, and other variables for your specific application needs. (see ad in this issue)

National Electrostatics Corp. \#U711

7540 Graber Road

P.O. Box 620310

Middleton, WI 53562-0310

Contact: Gregory A. Norton

Telephone: (608) 831-7600

Fax: (608) 256-4103

E-mail: nec@well.com

National Electrostatics Corp. manufactures multi-million volt ion beam systems for materials analysis and modification. These systems include dedicated instruments for RBS, PIXE, hydrogen profiling and other $\mathrm{MeV}$ ion beam techniques. Related ion sources, components and subsystems are also available.

(see ad in this issue)

Neocera, Inc. \#U953

10000 Virginia Manor Road Suite 300

Beltsville, MD 20705-4215

Contact: Tracy Fortuna

Telephone: (301) 210-1010

Fax: (301) 210-1042

Specialists in pulsed laser

deposition (PLD) and advanced thin film technologies will be exhibiting and demonstrating a fully operational PLD laboratory. Also displayed will be different PLD accessories and custom metal oxide films deposited on a variety of substrates. Literature regarding our $R \& D$ efforts, products, and services will be available.

$\$$ New Focus, Inc. \#U907

2630 Walsh Avenue

Santa Clara, CA 95051-0905

Contact: Milton Chang

Telephone: (408) 980-8088

Fax: (408) 980-8883

E-mail: contact@NewFocus.com Award-winning building blocks for absorption, FM, and nonlinear spectroscopy featuring tunable diode lasers, multi-pass absorption cells, differential optical receivers, modulators, ultra high-speed photodetectors and amplifiers. Our extensive range of laser tools includes ultrastable positioners, new tiny positioners, waveplates, polarizers, optics, and compact vacuum compatible picomotors to motorize your experiments. (see ad in this issue)

\section{Nicolet Instrument Corporation} \#A13

5225 Verona Road

Madison, WI 53711

Contact: Katie Stahl

Telephone: (608) 276-6345

Fax: (608) 273-5045

Nicolet will exhibit our research FT-IR spectrometer, the MagnaIR 850 . The 850 provides advanced DSP-driven electronics and firmware for collection of step scan, kinetics and rapid scan data, and a spectral range from $25,000-50 \mathrm{~cm}-1$. Nicolet's
FT-Raman Accessory Module will also be shown. The FT-

Raman accessory allows both IR and Raman data collection, processing and searching from one system.

\section{Omicron Associates \#U303}

1738 N. Highland Road,

Suite G101

Pittsburgh, PA 15241

Contact: Peter H. Sales

Telephone: (412) 831-2262

Fax: (412) 831-9328

OMICRON is a highly specialized materials research technology company offering equipment dedicated to surface science. Products include the world's widest range of UHV Scanning Probe Microscopes including Variable Temperature/5 Kelvin STM's and the state-of-the-art UHV STM SEM/SAM system for structural and chemical analysis. To further extend our range of quality components and strengthen our complete turn-key analytical system program, a new division "OMICRON ELECTRON

SPECTROSCOPY Ltd." adds a comprehensive range of high performance analyzers for XPS, AES, UPS, ARUPS, and HREELS.

Oxford Applied Research \#W77 Crawley Mill

Witney

Oxon OX8 5TJ

United Kingdom

Contact: Christian Bradley

Telephone: (44) 1993-773575

Fax: (44) 1993-702326

E-mail: sales@oxfordar.demon.co.uk

Manufacturers of scientific instruments for thin film research. Our range of RF and thermal crackers facilitate: GaN growth: ZnSe p-doping: in situ substrate cleaning: Atomic As, $\mathrm{Se}, \mathrm{S}$ for doping and bulk material growth: Oxidation. Other products include RHEED, mini e-beam evaporators, magnetrons, focused scanning ion and electron guns for SIMS, cleaning and beam neutralization.

Oxford Instruments, Inc. \#U508 130 A Baker Avenue

Concord, MA 01742

Contact: John Stephens

Telephone: (508) 369-9933

Fax: (508) 369-6616

Oxford Instruments presents MagLab Measurement Probes, a range of inserts designed to enhance experimental flexibility within MagLab "ultimate sensitivity" systems for materials characterization. Also shown are Teslatron measurement systems and the new Teslatron Probe System, with transport, thermal and susceptibility inserts. Cryostats for optical microscopy and spectroscopy will be available. Oxford Instruments, Inc., Microanalysis Group is a leading instrumentation company, offering innovative microanalytical technolo- gies. Intuitive Windows software, superior throughput, and easy data management make the Link ISIS and Link GEM the most popular microanalysis in the world.

(see ad in this issue)

Oxford University Press \#U205

198 Madison Avenue

New York, NY 10016

Contact: Jan Arrigo

Telephone: (212) 726-6200 ext. 6063

Fax: (212) 726-6441

Oxford University Press pub-

lishes a wide range of scholarly monographs, practical hand-

books and useful texts in

Materials Science.

\section{$\$$ Park Scientific Instruments} \#U812-813

1171 Borregas Avenue

Sunnyvale, CA 94087

Contact: David Campbell

Telephone: (408) 747-1600

Fax: (408) 747-1601

E-mail: Campbell@park.com

Park Scientific Instruments presents a complete family of award-winning Scanning Probe Microscopes for use in industry and research. Park Scientific Instruments will display

AutoProbe M5, the first SPM to offer both atomic resolution and large sample capacity in one instrument, AutoProbe $\mathrm{CP}$, an affordable, full featured SPM that incorporates fully motorized tip to sample approach and prealigned cantilever assemblies, and the IR-100 award winning AutoProbe VP for

UHV AFM, NC-AFM

Philips Electronic Instruments Co. \#U800-801

85 McKee Drive

Mahwah, NJ 07430

Contact: Lisa Schroeder

Telephone: (201) 529-6246

Fax: (201) 529-5084

Philips Electronic Instruments Company is the leading manufacturer of X-Ray Diffraction and

$X$-Ray Fluorescence equipment as well as a full ine of Scanning and Transmission Electron Microscopes. Information is available on all product lines. Philips Electronic Instruments Company is ISO 9001 certified. We adhere to the most stringent of the three ISO classifications which requires an established, effective quality system be in place.

(see ad in this issue)

Philips Semiconductors/ Materials Analysis Group \#U963

811 E. Arques Avenue, MS 65

Sunnyvale, CA 94088

Contact: Alan Morgan

Telephone: (408) 991-4868

Fax: (408) 991-4801

E-mail:morgana@scs.philips.com

Analytical services laboratory offering SIMS, GDMS, FIB, Auger, ESCA, RBS, AFM/STM, TEM, FESEM, EDX, XRF, XRD, Raman, FTIR, UV/Vis, GC/ MS/IR, GPC, ICP, AA, IC,

TGA/TMA/DSC, and acoustic microscopy for surface, interface, particle, thin film and bulk materials characterization. Trace element detection, high-resolution imaging and depth profiling, and precision cross-sectioning.

$\$$ Physical Electronics \#U807-808 6509 Flying Cloud Drive Eden Prairie, MN 55344

Contact: Molly Thuma

Telephone: (612) 828-6100

Fax: (612) 828-6322

Physical Electronics (PHI) develops, manufactures and markets a complete line of surface analysis instrumentation surface analysis subsystems, components and ultrahigh vacuum equipment. Surface analysis instruments from PHI utilize the Auger, ESCA / XPS and TOF-SIMS techniques. Vacuum products include ion pumps, gauge controllers and ion pump power supplies.

Plasma Sciences, Inc. \#A3 $7200 \mathrm{~A}$ Telegraph Sq. Drive Lorton, VA 22079

Contact: Steven Collins Telephone: (703) 550-7888

Toll-free: (800) 207-9776

Fax: (703) 339-9860

Plasma Sciences, Inc. specializes in the manufacture of high quality planar magnetron thin film deposition systems for research and pilot production. Multiple source DC/RF sputtering systems with recipe drive PC control are available as well as manual cost effective sputtering systems for smaller development applications. Other products include $R \& D$ scale RIE and reactive plasma etchers. 
Bockris and S. Kahn; Symmetry Through the Eyes of a Chemist by M. Hargittai and I. Hargittai; Principles of Polymer Chemistry by J. Ravve; Structural Electron Crystallography by D. Dorset; Introduction to High Temperature Superconductivity by $\mathrm{T}$. Sheahen; Case Studies in Superconducting Magnet Technology by Y. Iwasa; Stability of Superconductors by P. Dressner: Scanning Electron Microscopy and X-Ray Microanalysis by J. Goldstein et al.; Electron Microdiffraction by J. Spence; Plasmas and Polymers; Journal of Chemical Crystallography; Journal of Computer Assisted Microscopy; Journal of Environmental Polymer Degradation; Journal of Science Education and Technology; and Journal of Materials Synthesis and Processing.

Princeton Gamma-Tech, Inc. \#U815-816

1200 State Road

Princeton, NJ 08540

Contact: Jeannie Weakleim

Telephone: (609) 924-7310

Fax: (609) 924-1729

E-mail: jeannie@pgt.com

Microanalysis Systems for SEM,

(S) TEM and TEM: Si and Ge

detectors with PGT's patented

Digital Pulse Processor for

better X-ray data in less time.

Highlighted is the large $60 \mathrm{~mm}^{2}$ area PRISM for optimized analysis on field emission microscopes. Comprehensive $X$-ray analysis software and EM control in a multi-tasking environment. Computer-aided applications for EM and light microscopy include particle size and shape analysis, metallography, fractography, coatings thickness measurement, critical dimension measurement, stereo depth measurement and microscope automation.

(see ad in this issue)

Princeton Instruments, Inc. \#U961 3660 Quakerbridge Road

Trenton, NJ 08619

Contact: Sandy Kapica

Telephone: (609) 587-9797

Fax: (609) 587-1970

Princeton Instruments will show its complete line of systems for optical and X-ray spectroscopy and imaging. Systems include the widest selection of CCD based detectors and cameras, plus the industry's only fiber-coupled image intensified units. Systems offer single photon sensitivity, up to $4 \times 4$ pixels, and up to $85 \%$ quantum efficiency.

\section{Princeton Research Instruments} \#U809

P.O. Box 1174

Princeton, NJ 08542

Contact: Charles A. Crider

Telephone: (609) 924-0570

Exhibiting a full range of Reverse View LEED equipment including 5 RVL models ( 2 moveable and 3 fixed) and the new CVL8-120 conven- tional/convertible view LEED optics which is upgradable to reverse view. All optics operate with the PRI 11-020 LEED electronics. Our PRI RHEED system will also be shown as well as the complete PRI offering of invacuum stepper motors, translation stages, drive electronics, and accessories.

\section{Princeton Scientific Corp.} \#U968

P.O. Box 143

Princeton, NJ 08542

Contact: Hank Gerwers

Telephone: (609) 924-3011

Fax: (609) 924-3018

E-mail: PrinceScie@aol.com

Princeton Scientific Corp. offers various metal and oxide single crystals, like $\mathrm{W}, \mathrm{Mo}, \mathrm{Au}, \mathrm{SrTiO}_{3}$, $\mathrm{TiO}_{2}, \mathrm{MgO}$, etc. Cutting and polishing services are also provided. Also on display is a "precision wire saw" which allows for a very accurate cutting process and yields surfaces with almost 'lapped' quality.

Pure Tech, Inc. \#U505

Commerce Drive

P.O. Box 1319

Carmel, NY 10512

Contact: Matthew Willson

Telephone: (914) 878-4499

Fax: (914) 878-4727

Pure Tech is an ISO 9002

Certified American manufacturer of high purity materials for sputtering and evaporation. Pure Tech produces both standard and custom materials for $R \& D$ as well as production applications. In house capabilities include vacuum melting, inert gas hot pressing, metal and ceramic machining, custom designed backing plates, target bonding, and analytical services.

Ouantum Design \#U610

11578 Sorrento Valley Road

San Diego, CA 92121

Contact: Bill Zoeckler

Telephone: (619) 481-4400

Fax: (619) 481-7410

E-mail: blz@QUANDSN.com Quantum Design will be displaying its Magnetic Property Measurement System (MPMS) and Physical Property

Measurement System (PPMS).

The MPMS is the industry standard for ultra-sensitive magnetic measurements. The PPMS offers a turn-key platform on which to run a number of different experiments under full automation. Stop by and see our latest product advancements in expanding the capabilities of the MPMS and PPMS. (see ad in this issue)

\section{Quesant Instrument Corporation} \#U967

28038 Dorothy Drive

Agoura Hills, CA 91301

Contact: George C. McMurtry

Telephone: (818) 597-0311

Fax: (818) 991-5490

Quesant offers a full line of (QScope ${ }^{\mathrm{TM}}$ ) scanning probe microscopes starting at under $\$ 30,000$. All QScopes are research quality instruments that have been designed for ease of use and feature ScanAtomic ${ }^{\mathrm{TM}}$ control and analysis software operating under Windows ${ }^{\mathrm{TM}}$, Modulite ${ }^{\mathrm{TM}}$ (modulated laser mode) and the Isotopic Focal System ${ }^{\mathrm{TM}}$ (large sample capability and laser tracking).

(see ad in this issue)

\section{Research \& PVD Materials Corporation \#U906}

P.O. Box 4796

Wayne, NJ 07474-4796

Contact: Melvin J. Hollander

Telephone: (201) 575-4245

Fax: (201) 575-6490

RHK Technology, Inc. \#W63

1750 West Hamlin Road

Rochester Hills, MI 48309

Contact: John M. Green

Telephone: (810) 656-3116

Fax: (810) 656-8347

E-mail: green@rhk-tech.com RHK Technology, Inc. manufactures the most complete line of high performance Scanning Probe Microscopy (SPM) Control Systems and Accessories for the research scientist. Demonstrations on our Silicon Graphics and Personal Computer Control Systems will be conducted throughout the show. Also on display will be our Model UHV 300 Variable Temperature UHV STM Scan Head, our Nanonics NSOM products and our line of SPM components and accessories.

$\rightarrow$ Rigaku/USA, Inc. \#U901

Northwoods Business Park

199 Rosewood Drive, Suite 190

Danvers, MA 01923

Contact: Alan Robson

Telephone: (508) 777-2446

Fax: (508) 777-3594

E-mail: Rigaku@AOL.COM Rigaku will exhibit the new compact rotating anode X-ray generator. Combined with a new range of accessories, this generator forms the basis of a complete system for materials analysis. Specifically, accessories for the analysis of thin films, texture, stress and reflectometry are among those available.

RJ Lee Instruments, Ltd. \#U503 515 Pleasant Valley Road Trafford, PA 15085

Contact: David A. Crawford

Telephone: (412) 744-0100

Fax: (412) 744-0506

RJ Lee Instruments, Ltd. manufactures the PERSONAL SEM®, an applications-oriented, all digital scanning electron microscope, affordable, yet high in value and functionality with "point and click" operation; distributes SPECS GmbH surface analysis systems and components including the EA10+

Energy Analyzer, IQE 12/38 Ion Source, and UVS 10/35 Source.

-Schumacher \#U300

1969 Palomar Oaks Way

Carlsbad, CA 92009

Contact: Dorothy Foley

Telephone: (619) 929-6201

Fax: (619) 931-7819

Schumacher is a leading supplier of ultra-high purity chemicals and chemical delivery equipment to the semiconductor industry. In addition to our standard line of production chemicals for diffusion, oxidation and CVD processes, Schumacher also supplies precursors for CVD aluminum (DMAH, DMEAA), copper (CupraSelect(B), titanium nitride (TDMAT, TDEAT) and

high and low $\varepsilon$ dielectrics.

Scientific Instruments, Inc. \#A5

$4400 \mathrm{~W}$. Tiffany Drive

West Palm Beach, FL 33407

Contact: R. Michael Capers

Telephone: (407) 881-8500

Fax: (407) 881-8556

The revolutionary GENESIS

TCS, Temperature Control System will be featured. This Temperature Controller offers PC display and operation under Windows $^{\mathrm{TM}}$ or as a LabView ${ }^{\mathrm{TM}}$ Driver. Additionally, there is a remote Touch Screen Display Module provided as optional equipment. Information on varied cryogenic temperature sensors will also be available.

Scintag, Inc. \#A11

707 Kifer Road

Sunnyvale, CA 94086

Contact: Elisabeth Rippstein

Telephone: (408) 737-7200 ext. 104

Fax: (408) 737-9841

E-mail:76105.1241@

compuserve.com 
instrumentation, including configurations for phase identification, quantitative analysis, and single-crystal molecular structure determination. Specialized equipment developments include high-resolution optics for analyzing epitaxial materials, two-dimensional detectors for texture analysis, small angle scattering, and microdiffraction; and advanced optics to increase X-ray flux.

SKION Corporation \#U918 612 River Street

Hoboken, NJ 07030

Contact: Seong I. Kim

Telephone: (201) 216-5633

Fax: (201) 216-8929

E-mail: m1son@

vaxc.stevens-tech.edu

SKION is introducing the

world's first Solid State Ion

Beam Technology ${ }^{\mathrm{TM}}$ and Direct

Metal Ion Beam Deposition Equipment ${ }^{\mathrm{TM}}$. The state-of-theart Solid State Ion Beam Technology ${ }^{\mathrm{TM}}$ enables one to operate various metal ion sources while maintaining high vacuum and also providing superior thin film quality control over a large area at a very economic cost.

Solartron Instruments, Inc. \#U964

11261 Richmond Avenue

Houston, TX 77082

Contact: Mary A. Betts

Telephone: (713) 558-4930

Fax: (713) 558-4701

E-mail:100431.3503@

compuserve com

Solartron Frequency Response and gain-phase analyzers set the worldwide standard for quality and reliability in impedance measurement for electrochemistry and materials characterization. Solartron also manufactures high-speed potentiostats with uniform frequency response in all ranges for impedance, $\mathrm{DC}$ electrochemistry and EC noise analysis with the most advanced software available.

\SOPRA, Inc. \#U951

33 Nagog Park

P.O. Box 2619

Acton, MA 01720-6619

Contact: Alain Bourdon

Telephone: (508) 263-2520 ext. 114

Fax: (508) 263-2790

E-mail: sopra@delphi.com

The GESP5 is the first commercially available instrument to combine spectroscopic ellipsometry with accurate measurement of light scattering transmittance and reflectance as a function of wavelength, incidence angle, and polarization.
South Bay Technology, Inc. \#A1-2

1120 Via Callejon

San Clemente, CA 92673

Contact: David Henriks

Telephone: (800) 728-2233

Fax: (714) 492-1499

E-mail: 73531.1344@

compuserve com

South Bay Technology, Inc. will

be exhibiting the industry's most advanced sample prepara-

tion systems and supplies.

Featured will be systems for:

Orienting, Cutting \& Polishing

Single Crystals; Ultra-precise

thinning of cross-section TEM

samples; Selective etching of

GaAs/AIGaAs Hetero-

structures; and Damage free

cutting and polishing of soft

single crystals. Featured prod-

ucts include: IV3 Research

Grade Ion Milling System for

TEM, SEM and Optical

Microscopy; Tripod Polisher ${ }^{\mathrm{TM}}$

for TEM and SEM Polishing;

900 series Lapping and

Polishing Systems; and Real-

Time Back Reflection Laue

Camera. Application engineers

will be on hand to help you

solve your most difficult sam-

ple preparation problems.

Springer-Verlag New York, Inc. \#Ü210

175 Fifth Avenue

New York, NY 10010

Contact: Kenneth Quinn

Telephone: (212) 460-1577

Toll-free: (800) 777-4643 Ext. 577

Fax: (212) 533-5587

E-mail: kquinn@springer-ny.com

World Wide Web

http://www.springer-ny.com

Major new and best-selling

titles on display include $Y u$ and

Cardona's Fundamentals of

Semiconductor Physics, the

second edition of Ibach and

Lüth's Solid State Physics,

Chakraborty and Pietilainen's

The Quantum Hall Effects, the

third edition of Lüth's Surfaces and Interfaces of Solids, and the second edition of Mönch's Semiconductor Surfaces and Interfaces. Stop by to see these plus perennial best sellers - all available at $20 \%$ off list prices.

Staib Instruments, Inc. \#U403 813 Diligence Drive, Suite 121E Newport News, VA 23606 Contact: Lillyan Dylla

Telephone: (804) 873-0099

Fax: (804) 873-0130

Staib Instruments manufactures compact, high performance electron-optical equipment for in-situ materials analysis, including: RHEED and RHEED Vision to study structure and quality of thin films; Photo Emission Electron Microscopy (PEEM), a new technique for dynamic studies of chemical distributions with high time and spacial resolution; and Auger spectrometers for analytical surface studies.
Structure Probe, Inc./SPI Supplies \#U915

P.O. Box 656

West Chester, PA 19381-0656

Contact: Charles A. Garber

Telephone: (610) 436-5400

Toll-free: (800) 2424-SPI

Fax: (610) 436-5755

E-mail: StructPro@aol.com or

SpiSupp@aol.com

Structure Probe, Inc.: Independent laboratory providing nnovative electron microscopy and surface analysis services for solving materials science problems including HTC materials, diamond coatings and complex polymer systems. SPI Supplies: Sample preparation instruments and consumable supply items for electron microscopy and surface analysis laboratories. Exclusive SPI-Module ${ }^{\mathrm{TM}}$ Sputter/Carbon Coating System, Plasma Prep II ${ }^{\mathrm{TM}}$ etcher/asher, Plasma Prep $X^{\mathrm{TM}}$ Parallel Plate all solid state Plasma Etcher for anisotropic etching and "Tacky Dot" slides. See us on the WWW at http:/ / mail.cccbi.chester.pa.us/spi/spi home.html

Superconductive Components/ Target Materials, Inc. \#A10

1145 Chesapeake Avenue

Columbus, $\mathrm{OH} 43212$

Contact: J.R. Gaines

Telephone: (614) 486-0261

Fax: (614) 486-0012

E-mail: JRGSCI@aol.com

TMI manufactures high quality sputtering targets for Research and Industrial applications. Our product offering includes materials for FPD, Photovoltaic, Resistor, Hardness and other coating applications. Our parent company, $\mathrm{SCl}$, specializes in High $\mathrm{T}_{c}$ Superconductors as powders, targets, thin films, and Levitators. Stop by our booth for a demonstration of our new Melt Processed YBCO.

Sycon Instruments, Inc. \#U506 6757 Kinne Street

East Syracuse, NY 13057

Contact: Gwen Stell

Telephone: (315) 463-5297

Fax: (315) 463-5298

Sycon Instruments, Inc. will dis play its complete line of Thin

Film Deposition Monitors and Controllers utilizing the quartz crystal sensing techniques. A complete line of $\mathrm{HV}$ and UHV sensors and shutters for these products will also be displayed.

A deposition monitor based on the principle of Atomic Absorption will also be displayed for continuous monitoring of film deposition. A multipocket E-Beam Source Indexer for the control of $4 \times 6$ pocket E-Beam Guns will also be displayed.

Taylor \& Francis \#U111 1900 Frost Road, Suite 101 Bristol, PA 19007

Contact: Michelle Edwards Telephone: (215) 785-5800 ext. 15 Fax: (215) 785-5515

E-mail: medwards@tandfpa.com Taylor \& Francis is an international publisher and distributor of scientific, technical, and professional books and journals in physics, fiber optics, optoelectronics and other related disciplines. Titles include Philosophical Magazine, International Journal of Optoelectronics and Liquid Crystals.

TEC \#U917

10737 Lexington Drive

Knoxville, TN 37933

Contact: Lise Bender

Telephone: (615) 966-5856

Fax: (615) 675-1241

TEC specializes in fast, accurate stress measurements that help companies gauge how manufacturing and rework processes affect metallic and ceramic parts. We offer both products and lab services for measuring residual and loading stresses, and retained austenite content in steels.

- Telemark \#U406

51 Whitney Place

Fremont CA 94539

Contact: Gerald G. Henderson

Telephone: (510) 770-8700

Fax: (510) 770-8879

Telemark produces a wide range of PVD Components Our E-Beam Sources range from

$1.5 \mathrm{cc}$ to $345 \mathrm{cc}$ and our EB supplies cover the band from $3 \mathrm{~kW}$ to $30 \mathrm{~kW}$. We also offer multi wavelength instruments for plasma analysis, thin film monitors for reflection, transmission, or color measurement, and a quartz crystal deposition controller

Tencor Instruments \#U603

2400 Charleston Road

Mountain View, CA 94043

Contact: Judy Elliott

Telephone: (415) 969-6767

Fax: (415) 968-9482

Surface profiling systems for a variety of applications and budgets, providing comprehensive surface analysis on even very soft films. Guaranteed repeatability ensures highly accurate measurements. Large sample 
TexSEM Laboratories, Inc. \#U607

226 West 2230 No. \#120

Provo, UT 84604

Contact: John Bennion

Telephone: (801) 344-8990

Fax: (801) 344-8997

E-mail: 73251.1424@

compuserve.com

TSL will exhibit Orientation

Imaging Microscopy ${ }^{\mathrm{TM}}$

(OIMTM), a revolutionary new technique for analyzing local texture and grain boundary structure of polycrystalline materials. Thousands of backscatter Kikuchi diffraction patterns are captured and processed within minutes, linking local lattice orientation with grain morphology. Results are displayed intuitively via powerful image processing software.

Textron Defense Systems \#U400-401

2385 Revere Beach Parkway Everett, MA 02149

Contact: R. E. Gannon

Telephone: (617) 381-4630

Fax: (617) 381-4160

TDS has developed an affordable instrumentation package, LaserWave ${ }^{\mathrm{TM}}$, for the remote measurement of changes in material properties as a function of changes in the material's ultrasound characteristics. Thus, real time changes in surface or bulk properties, i.e. phase continuity, microstructure, hardness, etc. can be measured without physical contact of the material.

- Thermionics Laboratory, Inc. \#U810-811

22815 Sutro Street

P.O. Box 3711

Hayward, CA 94540

Contact: Mark Trujillo

Telephone: (510) 538-3304

Fax: (510) 538-2889

Thermionics manufactures vacuum systems, components, and hardware for all vacuum applications, including: ion pumps, titanium sublimators; $X Y Z$ manipulators, sample handling and transfer devices, differentially pumped rotary seals; gate, angle, and all-metal valves; 3-20 kW e-Gun ${ }^{\mathrm{TM}}$ evaporation sources and power supplies; feed throughs, gauges and controls, flanges, fittings; surface science, deposition, PLD, RHEED, and custom systems; DRS $^{\mathrm{TM}}$, an in-situ, remote substrate temperature measure ment/control system.
TopoMetrix \#U956

5403 Betsy Ross Drive

Santa Clara, CA 95054

Contact: Rich Fiore

Telephone: (717) 639-1210

Fax: (717) 639-5521

E-mail: 74132,3040@

compuserve.com

TopoMetrix manufactures the

TMX 2000, the world's most complete range of scanning probe microscope (SPM) and offers the widest range of scanning modes. Microscopes include Aurora ${ }^{\mathrm{TM}}$ NSOM (nearfield scanning optical microscope), Observer ${ }^{\mathrm{TM}}$ SPM for SEM, Voyager ${ }^{\mathrm{TM}}$ automated semiconductor-wafer SPM, and Accurex ${ }^{\mathrm{TM}}$ Discoverer ${ }^{\mathrm{TM}}$ and Explorer ${ }^{\mathrm{TM}}$ research grade SPMs with TrueMetrix ${ }^{\mathrm{TM}}$ closed loop

linearization for calibrated quantitative measurements.

Union Carbide Crystal Products \#S80

750 South 32nd Street

Washougal, WA 98671

Contact: Jack Mellone

Telephone: (360) 835-9812

Fax: (360) 835-9848

Crystal Products, located in the Pacific Northwest, offers Czochralski-grown Sapphire substrates for blue LED, superconductors, and SOI, radiationhardened, IC devices. Sizes are $2^{\prime \prime}$ and $3^{\prime \prime}$ diameter, C-plane; $2^{\prime \prime}$ to $6^{\prime \prime}$ diameter, R-plane; and SOS epitaxial wafers. See our Sapphire Research Kits containing substrates oriented to $\mathrm{M}, \mathrm{A}$ $R$, and $C$ axes.

Varian Vacuum Products \#U900 121 Hartwell Avenue Lexington, MA 02173 Contact: Phil Ciaccio Telephone: (800) 926-3000 ext. 3118 Fax: (603) 382-3451

Varian Vacuum Products will exhibit a broad range of high vacuum equipment including the Macrotorr, maintenancefree ceramic-bearing turbo pump and the Starcell® Ion Pump. On display will be a dry turbo pump station, a model 956 Turbo Leak Detector and the new senTorr ${ }^{\mathrm{TM}}$ Gauge Controller.

VAT, Inc. \#A6

500 West Cummings Park

Woburn, MA 01801

Contact: Sue Morin

Telephone: (617) 935-1446

Fax: (617) 935-3940

VAT will display a variety of viton-sealed and all-metal sealed vacuum valves for pump isolation, load-locks, downstream pressure control, beam lines and other applications. Patented VATSEALS, a simple method for metal sealing flat surfaced flanges in any shape, from $2^{\circ} \mathrm{K}$ to $300^{\circ} \mathrm{C}$, will also be shown

(see ad in this issue)
VCH Publishers, Inc. \#U108

220 East 23rd Street

New York, NY 10010-4606

Contact: Seth Ditchik

Telephone: (212) 683-8333

Fax: (212) 481-0897

E-mail: order@vch.com

International publisher of research monographs, reference sets, textbooks, and journals in the fields of materials science, physics, chemistry and chemical engineering, food science, and life sciences. New and featured titles include: Advanced Materials; Materials Science and Technology; Statistical Mechanics of Phases, Interfaces and Thin Films: Polymerization Process Modeling; Electrochromism; OneDimensional Metals; Materials Science of Microelectronics; Scanning Tunneling Microscopy and Spectroscopy; Sensors; and Transition Metal Oxides.

Virginia Semiconductor, Inc. \#U806

1501 Powhatan Street

Fredericksburg, VA 22401

Contact: N. Perry Cook

Telephone: (703) 373-2900

Fax: (703) 371-0371

(see ad in this issue)

Voltaix, Inc. \#U605

197 Meister Avenue

P.O. Box 5357

North Branch, NJ 0887

Contact: Debra A. Wagner

Telephone: (908) 231-9060

Fax: (908) 231-9063

Voltaix manufactures and distributes gases used for CVD and implant applications, including Diborane, Germane, Trimethylboron, and Methylsilane. These gases, as well as Silane, Phosphine, Silicon and Germanium Tetrafluoride and Boron Trifluoride, are available as pure gases and in a variety of mixtures. Examples of new applications for our products include plasma deposited dry processable photoresist based on Methylsilane and non-massselective boron implantation using boron-11 Diborane/

Hydrogen mixtures.

(see ad in this issue)

Iohn Wiley \& Sons, Inc. \#U110 605 Third Avenue

New York, NY 10158-0012

Contact: Beth Schacht

Telephone: (212) 850-6678

Fax: (212) 850-6264

E-mail: bschacht@jwiley.com

Please visit the Wiley booth and review our books and journals: Principles of Ceramics Processing, $2 E$ by James $\mathrm{S}$. Reed; Intermetallic Compounds, Volumes 1 and 2 edited by J. H. Westbrook and R. L. Fleischer; Principles of Plasma Discharges and Materials Processing by Michael A. Liberman and Allan \}.

Lichtenberg; and Phase

Transformations and Ablation in Laser-Treated Solids by Emil N. Sobol. Don't forget to pick up a complimentary copy of Mechanics of Composite Materials and Structures: An International Journal.

World Scientific Publishing Co., Inc. \#U107

1060 Main Street

River Edge, NJ 07661

Contact: Jo Anne Dingler

Telephone: (201) 487-9655

Fax: (201) 487-9656

E-mail: wspub@tigger.jvnc.net

Displaying: Fractal Growth

Phenomena 2nd edition

(Vicsek), Handbook of Solid

State Batteries and Capacitors

(Munshi), Sir Nevill Mott-

65 Years in Physics (Mott)

Synchrotron Radiation Sources-A Primer (Winick) and more high quality publications. Journal samples: Fractals, Innovations in Materials Research (IMR) and Surface

Review and Letters (SRL).

Meeting discount offered

X-Ray Optical Systems, Inc. \#U916

90 Fuller Road

Albany, NY 12205

Contact: J. Philip Bly

Telephone: (518) 442-5250

Fax: (518) 442-5292

E-mail: XOSPB@

CNSVAX.ALBANY.EDU

We design and fabricate both standard and custom capillary based optics for use with X-rays and neutrons. Options for focusing, collimating, and beam bending are available. These optics guide the beam using multichannel hollow capillary fibers. The X-rays or neutrons are transmitted via multiple total external reflections from the smooth inner walls of the hollow capillary channels.

Zygo Corporation \#U402

Laurel Brook Road

Middlefield, CT 06455

Contact: Polly White

Telephone: (203) 347-8506

Fax: (203) 346-4188

Zygo Corporation, a world leader in surface information technology and precision manufacturing, is exhibiting their NewView 100 and GPI interferometers. NewView 100 Surface Structure Analyzer provides 3D images and measurements on a wide range of materials and surfaces. GPI systems provide 